Colombo, C. (2010) Orbital dynamics of earth-orbiting 'smart dust' spacecraft under the effects of solar radiation pressure and aerodynamic drag. In: AIAA/AAS Astrodynamics Specialist Conference 2010, 2-5 Aug 2010, Toronto, Canada.

http://strathprints.strath.ac.uk/26358/

Strathprints is designed to allow users to access the research output of the University of Strathclyde. Copyright (C) and Moral Rights for the papers on this site are retained by the individual authors and/or other copyright owners. You may not engage in further distribution of the material for any profitmaking activities or any commercial gain. You may freely distribute both the url (http://strathprints.strath.ac.uk) and the content of this paper for research or study, educational, or not-for-profit purposes without prior permission or charge. You may freely distribute the url (http://strathprints.strath.ac.uk) of the Strathprints website.

Any correspondence concerning this service should be sent to The Strathprints Administrator: eprints@cis.strath.ac.uk 


\title{
Orbital dynamics of Earth-orbiting 'smart dust' spacecraft under the effects of solar radiation pressure and aerodynamic drag
}

\author{
Camilla Colombo ${ }^{1}$ and Colin McInnes ${ }^{2}$ \\ Advanced Space Concepts Laboratory, University of Strathclyde, Glasgow, UK, G1 IXJ
}

\begin{abstract}
This paper investigates how the perturbations due to asymmetric solar radiation pressure, in presence of Earth's shadow, and atmospheric drag can be balanced to obtain long-lived Earth centered orbits for swarms of SpaceChips, without the use of active control. The secular variation of Keplerian elements is expressed analytically through an averaging technique. Families of solutions are then identified where a Sun-synchronous apse-line precession is achieved passively. The long-term evolution is characterized by librational motion, progressively decaying due to the non-conservative effect of atmospheric drag. Therefore, long-lived orbits can be designed through the interaction of energy gain from asymmetric solar radiation pressure and energy dissipation due to drag. In this way, the short life-time of high area-to-mass spacecraft can be greatly extended (and indeed selected). In addition, the effect of atmospheric drag can be exploited to ensure the end-of life decay of SpaceChips, thus preventing long-lived orbit debris.
\end{abstract}

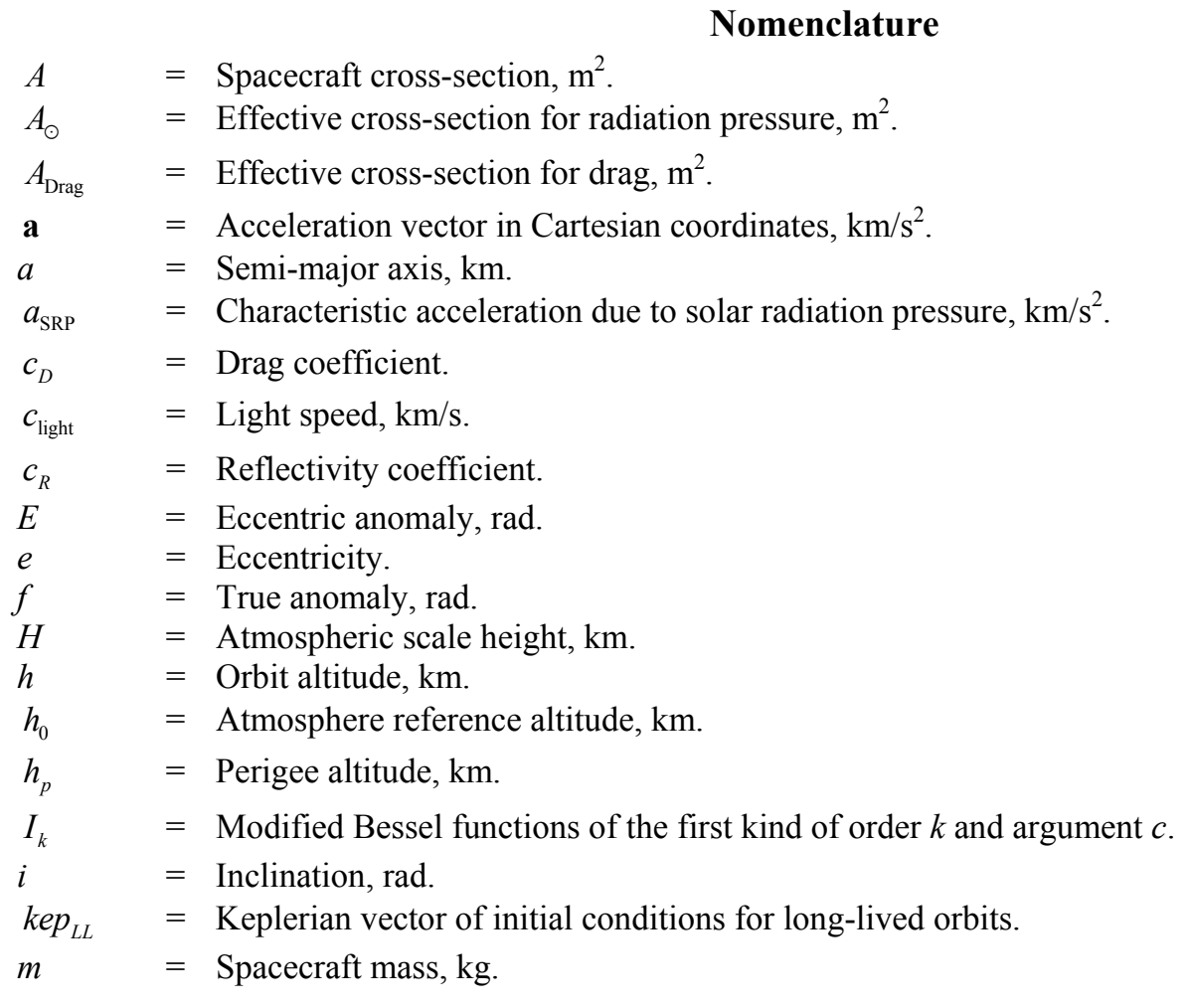

\footnotetext{
${ }^{1}$ Research Fellow Ph.D., Advanced Space Concepts Laboratory, Department of Mechanical Engineering, James Weir Building, camilla.colombo@strath.ac.uk; AIAA Member.

${ }^{2}$ Professor Ph.D., Advanced Space Concepts Laboratory, Department of Mechanical Engineering, James Weir Building, colin.mcinnes@strath.ac.uk; AIAA Member.
} 


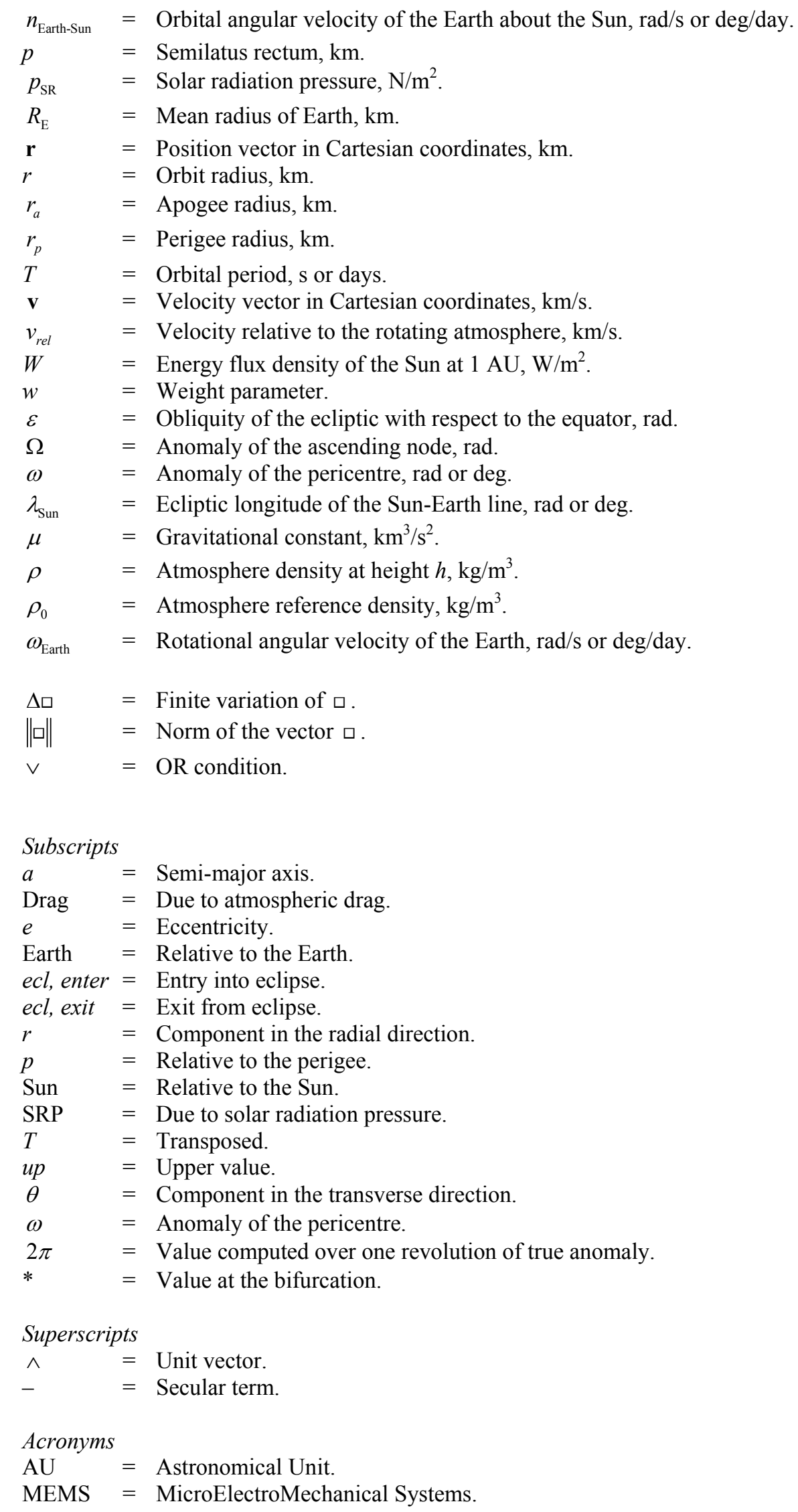




$\begin{array}{ll}\text { RHS } & =\text { Right-Hand Side. } \\ \mathrm{SRP} & =\text { Solar Radiation Pressure. } \\ \text { Constants } & \\ \mathrm{AU} & =149597870.7 \mathrm{~km} . \\ c_{\text {light }} & =299792.458 \mathrm{~km} / \mathrm{s} . \\ R_{\mathrm{E}} & =6378.16 \mathrm{~km} . \\ W & =1367 \mathrm{~W} / \mathrm{m}^{2} . \\ \varepsilon & =23.43928111 \mathrm{deg} . \\ \mu_{\text {Earth }} & =3.986004461921757 \times 10^{5} \mathrm{~km}^{3} / \mathrm{s}^{2} . \\ \mu_{\text {Sun }} & =1.3272448769 \times 10^{11} \mathrm{~km}^{3} / \mathrm{s}^{2} .\end{array}$

\section{Introduction}

Advances in miniaturization are enabling the development of small 'Smart Dust' devices with sensing, computing and communication capabilities. Current concepts for functional devices have been designed by exploiting existing capabilities, such as satellite-on-a-chip [1-2]. These developments offer the possibility of fabricating vast numbers of micro-spacecraft for use in swarm applications and with launch at low cost. Significantly smaller MEMS (microelectromechanical systems) devices with sensing, computing, bi-directional communication and micro-power sources are currently in laboratory development for terrestrial applications with a displaced volume of order $10 \mathrm{~mm}^{3}$ [3]. Atchison and Peck designed a passive, sun-pointing millimeter solar sail, whose bus is constituted by a $1 \mathrm{~cm}$ square and $25 \mu \mathrm{m}$ thick silicon microchip. The proposed design integrates solar cells and communications in a solid state device [4].

The deployment of vast numbers of such 'SpaceChips' will enable future missions, such as global sensor networks for Earth observation and communications, distributed space missions for multi-point, real-time sensing for space science (space weather, geomagnetic physics, reflectometry), interplanetary exploration in support of conventional spacecraft, or deployment in the vicinity of a large spacecraft for diagnostic or environmental detection purposes. The considerably smaller dimensions of SpaceChips envisage their deployment in orbit from a CubeSat or as piggy-back on a conventional spacecraft, thus allowing significant launch cost savings.

As an early example of a SpaceChip-scale swarm, project West Ford in 1963, placed a ring of $4.8 \times 10^{8}$ copper dipole antennas $(1.78 \mathrm{~cm}$ long needles, with a diameter of $17.8 \mu \mathrm{m})$ into orbit to allow global radio communication [5]. The motion of the individual dipoles, from dispensing to final re-entry through the atmosphere was modeled and observed.

The realization of these new concepts requires an understanding of orbital dynamics at extremes of spacecraft length-scale. The significantly higher area-to-mass ratio of such devices, with respect to conventional spacecraft, allows new insights into orbital dynamics since perturbations such as solar radiation pressure (SRP) and aerodynamic drag become dominant with respect to the Earth's gravity. Rather than counteracting these disturbances to the natural Keplerian motion, the interaction physics at small length-scales can be exploited and passive methods for orbit design can be envisaged without the use of active orbit control. This paper presents an analysis of longlived orbits for SpaceChips which uses the energy input from asymmetric solar radiation pressure to offset the energy dissipation of atmospheric drag.

The effect of natural perturbations on small particles has been studied extensively in the vast literature on the dynamics of cosmic dust in the solar system whose motion is perturbed by solar gravity, solar radiation pressure, Poynting-Robertson drag force, planetary oblateness and electromagnetic forces [6-10]. The effect of solar radiation pressure, zonal and tesseral harmonics of the Earth's gravitational potential, luni-solar third body perturbations, atmospheric drag on high area-to-mass ratio objects are also of particular interest to explain the long-term dynamical evolution of small debris particles released into Earth orbit. For example, orbital observations and high accuracy numerical integrations led to the discovery of a class of objects which remain in orbit for long durations due to the effect of solar radiation pressure in the synchronous and semi-synchronous orbital regime [11-12]. Furthermore, the effects of solar radiation pressure have been observed since 1960 in the orbital behavior of satellites such as the ECHO balloon [13], Vanguard [14] and many others [15] and is exploited as main propulsive force for solar sailing, when exerted on a large reflective membrane [16-17]. McInnes at al. showed that a small solar sail can be used to artificially process the apse line of an orbit, to provide sun-synchronous tracking of the geomagnetic tail [18]. 
Oyama at al. extended the analysis to the whole phase-space and defined the Hamiltonian of the system to investigate the evolution of the orbital elements [19]. Due to the characteristic of the orbit selected for the geomagnetic tail exploration mission, only solar radiation pressure was considered, and Earth eclipses were neglected; under these assumptions an equilibrium precessing orbit can be found analytically. De Juan Ovelar et al. explored the possible exploitation of the effects of solar radiation pressure on artificial nano-spheres and the design of a coating for spherical particles to engineer the ratio between the radiation pressure and the gravitational force. Earth-Mars transfer orbits were designed by using the particle coating as control parameter [20].

This paper investigates how the perturbations of solar radiation pressure and atmospheric drag can be balanced to obtain long-lived Earth centered orbits for swarms of SpaceChips, without the use of active control. Given the initial orbital elements of the spacecraft, the shadow geometry is determined as a function of semi-major axis, eccentricity and angular displacement between the Sun-Earth line $\lambda_{\text {Sun }}$ and the orbit pericentre $\omega$. The secular change of the in-plane orbital elements over a single orbit revolution is then evaluated analytically; an expression is derived for the variation of Keplerian elements due to solar radiation pressure, which takes into account the Earth's shadow through an analytic expression for the exit and entry true anomaly from/into eclipses. An analysis of the change in orbital elements due to orbital perturbations on such small devices is then performed. A search for initial orbital conditions for long-lived orbits is performed through a global search over a wide range of orbit eccentricities, altitudes of the pericentre and different values of $\omega-\lambda_{\text {Sun }}$, and local optimization through non-linear programming. The precession of the apse line of the orbit synchronously with the Sun is imposed in order to ensure the same conditions on the solar radiation pressure perturbation at each revolution.

From this initial analysis the paper presents families of long-lived orbits for swarms of SpaceChips where the condition of Sun-synchronous apse-line precession is achieved passively, without any propellant mass consumption. The conditions for long-lived orbits are then identified in the orbit phase-space and numerical integration of the secular variation of orbital elements is used to characterize the long-term evolution of the orbits. Different families of orbits are presented: in those regions of the phase-space where the effect of atmospheric drag is negligible, equilibrium orbits can be found under the effect of solar radiation only. If the initial condition is in a certain region around the equilibrium solution set, the long-term evolution is characterized by librational motion, progressively decaying due to the non-conservative effect of atmospheric drag. It is possible to recognize different arcs of the orbit evolution where the trajectory is dominated either by drag or by solar radiation pressure. Asymmetry in solar radiation pressure due to eclipses leads to modulation of the orbit energy and angular momentum, and families of orbits can be found where the energy gain due to radiation pressure balances the energy dissipation due to drag. A comparison with the drag-only and SRP-only scenarios is shown; the orbit lifetime and the final re-entry in the atmosphere are assessed.

Through the paper it is shown that the exploitation of the natural effects of solar radiation and atmospheric drag provides a means of enabling long-lived orbits for micro-spacecraft without the use of active control and ensures the final re-entry of the SpaceChip devices, so that they do not constitute future space debris. Finally, some applications for swarm of SpaceChip devices are proposed. The paper is organized as follows: Section II introduces the analytical approach to compute the orbit evolution; the initial conditions for long-lived orbits and the method adopted to identify them are described in Section III. Section IV and V present the solution for long-lived orbits and the long-term evolution under the effect of solar radiation pressure and drag. Finally some mission applications are proposed in Section VI.

\section{Orbit evolution}

A semi-analytical theory is used to compute the orbit evolution of the spacecraft under the influence of solar radiation pressure and drag. The secular variation of the orbital elements is obtained by averaging Gauss' planetary equations in the true anomaly or eccentric anomaly form. As a first approach, we consider only the solar radiation pressure and the atmospheric drag, whose perturbing accelerations are proportional to the area-to-mass ratio of the spacecraft; hence their effect becomes dominant for SpaceChips. Future work will include the influence of the Earth's oblateness, which causes a secular drift of the perigee and the argument of the ascending node. We constrain the present study to orbits lying on the ecliptic plane. In the remainder of this section, the semi-analytical model used for orbit propagation will be presented. 


\section{A. Secular variation of Keplerian elements due to solar radiation pressure}

We consider a spacecraft on an Earth-centered orbit lying in the ecliptic plane, with the geometry represented in Fig. $1^{\ddagger}$. The satellite is subjected to the acceleration due to solar radiation pressure given by

$$
\begin{aligned}
& a_{r, \mathrm{SRP}}=a_{\mathrm{SRP}} \cos \left(\omega-\lambda_{\mathrm{Sun}}\right) \\
& a_{\theta, \mathrm{SRP}}=-a_{\mathrm{SRP}} \sin \left(\omega-\lambda_{\mathrm{Sun}}\right)
\end{aligned}
$$

where $a_{r}$ and $a_{\theta}$ are the components of the acceleration $\mathbf{a}_{\mathrm{SRP}}=\left[\begin{array}{ll}a_{r, \mathrm{SRP}} & a_{\theta, \mathrm{SRP}}\end{array}\right]^{T}$ in the radial and transversal directions in the orbital plane, and the characteristic acceleration

$$
a_{\mathrm{SRP}}=\frac{p_{\mathrm{SR}} c_{R} A_{\odot}}{m}
$$

where $p_{\mathrm{SR}}$ is the solar pressure $p_{\mathrm{SR}}=\frac{W}{c_{\text {light }}}=4.56 \times 10^{-6} \frac{\mathrm{N}}{\mathrm{m}^{2}}$ (with $W$ the energy flux density of the Sun at 1 AU and $c_{\text {light }}$ the speed of light), $c_{\mathrm{R}}$ the reflectivity coefficient, $A_{\odot}$ is the area exposed to the Sun, which is considered constant in the following derivation, and $m$ is the mass of the satellite. The angle $\omega$ is the argument of the orbit perigee and the angle $\lambda_{\text {sun }}$ describes the direction of the Sun-line radiation (that is equal to the ecliptic longitude of the Sun minus $\pi$ ). Both $\omega$ and $\lambda_{\text {Sun }}$ are measured with respect to a fixed arbitrary direction, such that the angle $\omega-\lambda_{\text {Sun }}$ represents the angular displacement between the orbit pericentre and the Sun-line direction. Eq. (1) assumes that the Sun is at infinite distance from the satellite, that is the parallax of the Sun is negligible.

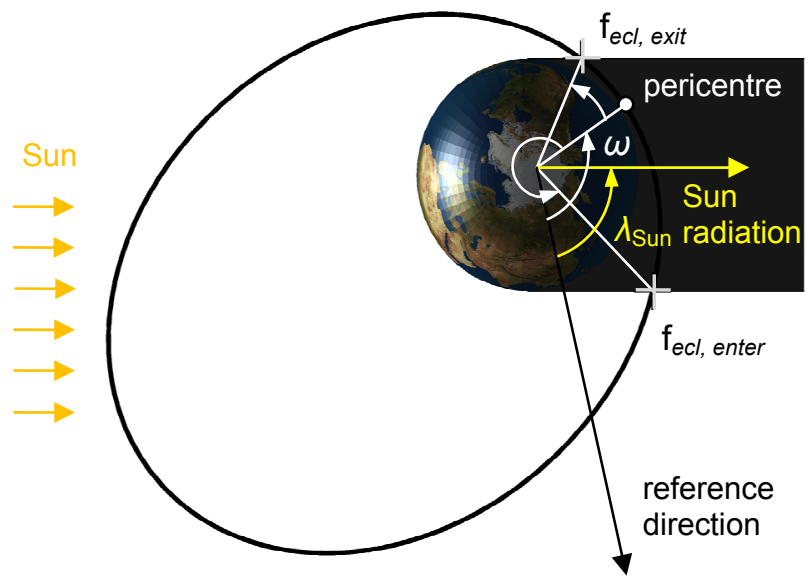

Fig. 1 Orbit geometry.

To investigate the effect of SRP on the spacecraft orbit, we consider the planar terms of the Gauss' equations, written as function of the true anomaly $f[21]$

\footnotetext{
* In the figures the acceleration due to SRP is shown directed along the horizontal axis but this does not introduce any simplification in the formulation, as the angles are measured from a reference arbitrary direction.
} 


$$
\begin{aligned}
& \frac{d a}{d f}=\frac{2 p r^{2}}{\mu\left(1-e^{2}\right)^{2}}\left(e \sin f \cdot a_{r}+\frac{p}{r} \cdot a_{\theta}\right) \\
& \frac{d e}{d f}=\frac{r^{2}}{\mu}\left(\sin f \cdot a_{r}+\left(1+\frac{r}{p}\right) \cos f \cdot a_{\theta}+e \frac{r}{p} \cdot a_{\theta}\right) \\
& \frac{d \omega}{d f}=\frac{r^{2}}{\mu e}\left(-\cos f \cdot a_{r}+\left(1+\frac{r}{p}\right) \sin f \cdot a_{\theta}\right)
\end{aligned}
$$

where $\mu$ is the gravitational constant of the Earth. $a, e$ and $\omega$ are the in-plane orbital elements, $f$ is the true anomaly, $r$ is the orbit radius, and $p$ the semilatus rectum. An averaging technique is used to compute the long-term variation of the orbital elements, i.e. Eqs. (3) are integrated in true anomaly, considering the other orbital elements to be constant over one orbit revolution. In the case of a constant disturbing acceleration in a fixed inertial direction defined in Eq. (1), Eqs. (3) can be solved in closed form to find the primitive functions $\mathrm{f}_{a}, \mathrm{f}_{e}, \mathrm{f}_{\omega}$ for semi-major axis, eccentricity and anomaly of the pericentre:

$$
\begin{aligned}
& \mathrm{f}_{a}\left(a, e, \omega-\lambda_{\text {Sun }}\right)=\int \frac{d a}{d f} d f+c_{a} \\
& \mathrm{f}_{e}\left(a, e, \omega-\lambda_{\text {Sun }}\right)=\int \frac{d e}{d f} d f+c_{e} \\
& \mathrm{f}_{\omega}\left(a, e, \omega-\lambda_{\text {Sun }}\right)=\int \frac{d \omega}{d f} d f+c_{\omega}
\end{aligned}
$$

Some integration constants $c_{a}, c_{e}$ and $c_{\omega}$ are introduced in the primitive functions but they are removed when the primitive function is evaluated at two limits of integration. Substituting Eq. (1) in Eqs. (3) the indefinite integrals can be computed, as shown in Eqs. (4). After some algebraic manipulations we obtain:

$$
\begin{aligned}
\mathrm{f}_{a}= & -\frac{2 a^{3}\left(1-e^{2}\right)}{\mu} a_{\mathrm{SRP}}\left(\frac{\cos \left(\omega-\lambda_{\mathrm{Sun}}\right)+e \sin \left(\omega-\lambda_{\mathrm{Sun}}\right) \sin f}{e(1+e \cos f)}\right)+c_{a} \\
\mathrm{f}_{e}= & \frac{a^{2}\left(1-e^{2}\right)^{2}}{\mu} a_{\mathrm{SRP}}\left(\sin \left(\omega-\lambda_{\mathrm{Sun}}\right)\left(-\frac{3}{2} \frac{E}{\left(1-e^{2}\right)^{3 / 2}}+\frac{\sin f\left(\cos f\left(-8 e^{4}+10 e^{2}-2\right)+6 e\left(1-e^{2}\right)\right)}{4\left(1-e^{2}\right)^{2}(1+e \cos f)^{2}}\right)+\right. \\
& \left.-\frac{\cos \left(\omega-\lambda_{\mathrm{Sun}}\right)}{2 e^{2}(1+e \cos f)^{2}}\left(1+2 e \cos f+e^{2}\right)\right)+c_{e} \\
\mathrm{f}_{\omega}= & -\frac{a^{2}\left(1-e^{2}\right)^{2}}{\mu e} a_{\mathrm{SRP}}\left(\cos \left(\omega-\lambda_{\mathrm{Sun}}\right)\left(\frac{3}{2} \frac{E}{\left(1-e^{2}\right)^{3 / 2}}-\frac{e \sin f}{\left(1-e^{2}\right)(1+e \cos f)}-\frac{1}{2} \frac{(e+\cos f) \sin f}{\left(1-e^{2}\right)(1+e \cos f)^{2}}\right)+\right. \\
& \left.+\sin \left(\omega-\lambda_{\mathrm{Sun}}\right) \frac{1+2 e \cos f}{2 e^{2}(1+e \cos f)^{2}}\right)+c_{\omega}
\end{aligned}
$$

where $E$ is the eccentric anomaly which is a bijective relation of the true anomaly

$$
E=2 \arctan \left(\sqrt{\frac{1-e}{1+e}} \tan \frac{f}{2}\right)
$$


Note that Eqs. (5) have been obtained under the assumption that the orbit lies on the ecliptic plane. Blitzer [22] follows the work by Cook [23] to obtain a more general form of these equations (dropping the assumption of a planar orbit), expressed as function of true anomaly and eccentric anomaly. Kozai [24] and Aksnes ${ }^{\S}$ [25] express the same variations as a function of the eccentric anomaly. The equivalence of Eqs. (5) to the general form in the literature can be verified by setting $\Omega=0$ and $i=\varepsilon$, that is the orbit inclination $i$ (with respect to the Earth equatorial plane) is equal to the obliquity of the ecliptic with respect to the Earth equator $\varepsilon$. Eqs. (5) assume that the disturbing acceleration $a_{\mathrm{SRP}}$ is constant when the spacecraft is in sunlight, i.e., the variation of the solar flux over time is neglected, and the exposed area $A_{\odot}$ in Eq. (2) is considered constant (i.e., the spacecraft has a spherical shape or the attitude of the spacecraft is kept constant with respect to the Sun direction). At this point, the total variation of the orbital elements can be evaluated over the orbit arc in which the spacecraft is in sunlight: $\left[0, f_{\text {ecl, enter }}\right],\left[f_{\text {ecl, exit }}, 2 \pi\right]$, which is function of $a, e, \omega-\lambda_{\text {Sun }}$ at the orbit pericentre and the argument of true anomaly at which the satellite enters and exits the eclipses, $f_{\text {ecl, enter }}$ and $f_{\text {ecl, exit }}$. Since we are assuming the orbital elements and $a_{\mathrm{SRP}}$ to be fixed over one orbit revolution, at their value at the pericentre, the total variation of the orbital elements can be written as:

$$
\begin{aligned}
& \Delta a_{\mathrm{SRP}, 2 \pi}\left(a, e, \omega-\lambda_{\mathrm{Sun}}, f_{\text {ecl, exit }}, f_{\text {ecl, enter }}\right)=\left[\mathrm{f}_{a}\right]_{0}^{f_{\text {ecl enter }}}+\left[\mathrm{f}_{a}\right]_{f_{\text {ecl, exit }}}^{2 \pi}=\left[\mathrm{f}_{a}\right]_{f_{\text {cel, exit }}}^{f_{\text {cel enter }}} \\
& \Delta e_{\mathrm{SRP}, 2 \pi}\left(a, e, \omega-\lambda_{\mathrm{Sun}}, f_{\text {ecl, exit }}, f_{\text {ecl, enter }}\right)=\left[\mathrm{f}_{e}\right]_{0}^{f_{\text {ecl, ener }}}+\left[\mathrm{f}_{e}\right]_{f_{\text {ccl, exit }}}^{2 \pi}=\left[\mathrm{f}_{e}\right]_{f_{\text {ccl, exit }}}^{f_{\text {ex, ener }}}
\end{aligned}
$$

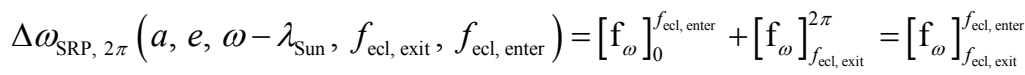

Note that, in the second formulation of the RHS of each of Eqs. (6), $2 \pi$ needs to be added to $f_{\text {ecl, enter }}$ and the corresponding $E_{\text {ecl, enter }}$ if $f_{\text {ecl, enter }}<f_{\text {ecl, exit }}$.

In the general three-dimensional case the limits of integration come from the solution of a $4^{\text {th }}$ order equation in $\cos f$ [26 pp. 155-162],[27 Sec. 2.5.1]. In the planar case we consider (i.e., the orbit lies in the ecliptic plane), the arguments of true anomaly when the spacecraft enters and leaves the Earth's shadow can be expressed as a closedform function of the orbital elements: $f_{\text {ecl, enter }}\left(a, e, \omega-\lambda_{\text {Sun }}\right), f_{\text {ecl, exit }}\left(a, e, \omega-\lambda_{\text {Sun }}\right)$. Assuming that the parallax of the Sun is negligible (see Fig. 1):

$$
\begin{array}{ll}
f_{\text {ecl, exit }}\left(a, e, \omega-\lambda_{\text {Sun }}\right): & \left\{\begin{array}{l}
r \cos \left(\omega-\lambda_{\text {Sun }}+f\right)>0 \\
r \sin \left(\omega-\lambda_{\text {Sun }}+f\right)=+R_{\mathrm{E}}
\end{array}\right. \\
f_{\text {ecl, enter }}\left(a, e, \omega-\lambda_{\text {Sun }}\right): & \left\{\begin{array}{l}
r \cos \left(\omega-\lambda_{\text {Sun }}+f\right)>0 \\
r \sin \left(\omega-\lambda_{\text {Sun }}+f\right)=-R_{\mathrm{E}}
\end{array}\right.
\end{array}
$$

where $R_{\mathrm{E}}$ is the mean radius of the Earth, and $r$ is the orbital radius. The expressions for $f_{\text {ecl, exit }}\left(a, e, \omega-\lambda_{\text {Sun }}\right)$ and $f_{\text {ecl, enter }}\left(a, e, \omega-\lambda_{\text {Sun }}\right)$ can be found analytically, after some algebraic manipulations and are given by

$$
\begin{aligned}
& f_{\text {ecl, exit }}=\mp \arccos \left[\frac{-R_{\mathrm{E}} d_{\text {exit }}+a\left(1-e^{2}\right) \cos \left(\omega-\lambda_{\text {Sun }}\right) \sqrt{\left(1-e^{2}\right)\left(a^{2}\left(1-e^{2}\right)-R_{\mathrm{E}}^{2}-2 a e R_{\mathrm{E}} \sin \left(\omega-\lambda_{\text {Sun }}\right)\right)}}{a^{2}\left(1-e^{2}\right)^{2}+e^{2} R_{\mathrm{E}}^{2}-2 a e R_{\mathrm{E}}\left(1-e^{2}\right) \sin \left(\omega-\lambda_{\text {Sun }}\right)}\right] \\
& f_{\text {ecl, enter }}=\mp \arccos \left[\frac{-R_{\mathrm{E}} d_{\text {enter }}+a\left(1-e^{2}\right) \cos \left(\omega-\lambda_{\text {Sun }}\right) \sqrt{\left(1-e^{2}\right)\left(a^{2}\left(1-e^{2}\right)-R_{\mathrm{E}}^{2}+2 a e R_{\mathrm{E}} \sin \left(\omega-\lambda_{\text {Sun }}\right)\right)}}{a^{2}\left(1-e^{2}\right)^{2}+e^{2} R_{\mathrm{E}}^{2}+2 a e R_{\mathrm{E}}\left(1-e^{2}\right) \sin \left(\omega-\lambda_{\text {Sun }}\right)}\right]
\end{aligned}
$$

\footnotetext{
$\S$ Aksnes also corrects some misprints present in Kozai’s paper.
} 
where we denote

$$
\begin{aligned}
& d_{\text {exit }}=e R_{\mathrm{E}}-a\left(1-e^{2}\right) \sin \left(\omega-\lambda_{\text {Sun }}\right) \\
& d_{\text {enter }}=e R_{\mathrm{E}}+a\left(1-e^{2}\right) \sin \left(\omega-\lambda_{\text {Sun }}\right)
\end{aligned}
$$

The $\mp$ sign in front of the right term of Eqs. (8) identifies the two symmetric positions which straddle the pericentre (and the line of apsides) as shown in Fig. 2. The " $x$ " symbol indicates the two possible solutions of the first of Eqs. (8) for $f_{\text {ecl,exit }}\left(f_{\text {ecl,exit }}^{-}\right.$and $f_{\text {ecl,exit }}^{+}$), whereas the "+" symbol indicates the two possible solutions of the second of Eqs. (8) for $f_{\text {ecl, enter }}\left(f_{\text {ecl, enter }}^{-}\right.$and $\left.f_{\text {ecl, enter }}^{+}\right)$. The superscript “+” indicates the solution with an angle between 0 and $\pi$, the superscript " - " indicates the solution with an angle between $\pi$ and $2 \pi$. An algorithm was developed to identify the correct solution of each of Eqs. (8) corresponding to the actual shadow boundary, by verifying whether the orbit pericentre or apocentre is in umbra.

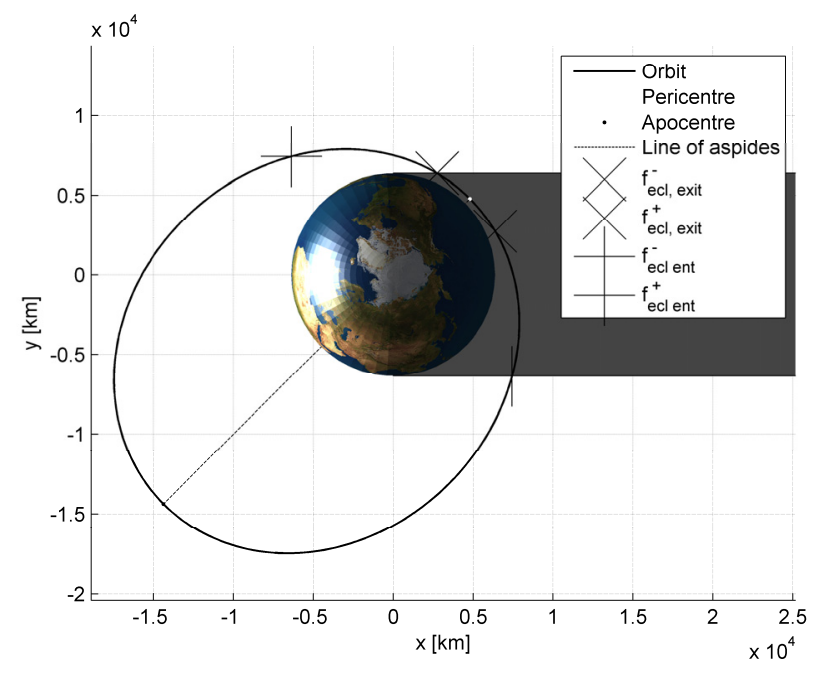

Fig. 2 Eclipse geometry: solutions of Eqs. (8). The " $x$ " symbol indicates the two possible solutions for the equation of the exit true anomaly; the "+" symbol indicates the two possible solutions for the equation of the entry true anomaly. The superscript "+" indicates the solution with an angle between 0 and $\pi$, the superscript "_" indicates the solution with an angle between 0 and $2 \pi$.

At this point, the expressions for $f_{\text {ecl, exit }}\left(a, e, \omega-\lambda_{\text {Sun }}\right)$ and $f_{\text {ecl, enter }}\left(a, e, \omega-\lambda_{\text {Sun }}\right)$ given by Eq. (8) can be substituted into Eqs. (6) to give the actual variation of orbital elements considering the Earth's shadow. If the spacecraft does not enter into eclipses, $f_{\text {ecl, enter }}=f_{\text {ecl, exit }}+2 \pi$, and some terms of Eqs. (6) vanish (the terms containing $\sin f$ and $\cos f$ ), in particular the variation of semi-major axis goes to zero. In the case of no eclipses, Eqs. (6) simplify to the formulation used by McInnes et. al [18] and Oyama et al. [19]. The secular and long-period rate of change of the orbital elements can be obtained by dividing Eqs. (6) by the Keplerian orbital period

$$
T=2 \pi \sqrt{\frac{a^{3}}{\mu}}
$$

thus giving 


$$
\begin{aligned}
& \frac{d \bar{a}}{d t}_{\mathrm{SRP}}=\frac{\Delta a_{\mathrm{SRP}, 2 \pi}}{2 \pi} \sqrt{\frac{\mu}{a^{3}}} \\
& \frac{d \bar{e}}{d t}_{\mathrm{SRP}}=\frac{\Delta e_{\mathrm{SRP}, 2 \pi}}{2 \pi} \sqrt{\frac{\mu}{a^{3}}} \\
& \frac{d \bar{\omega}}{d t}_{\mathrm{SRP}}=\frac{\Delta \omega_{\mathrm{SRP}, 2 \pi}}{2 \pi} \sqrt{\frac{\mu}{a^{3}}}
\end{aligned}
$$

where the superscript dash is used to indicate the secular variation. The line of apsides of the ellipse will rotate due to the perturbing solar radiation pressure acceleration, with a mean rate of precession given by the third of Eqs. (11). Moreover, if we consider uniform motion of the Earth around the Sun on a circular orbit (i.e. $\lambda_{\text {Sun }}(t)=\lambda_{\text {Sun }}(0)+n_{\text {Earth-Sun }} t$, we can express the third of Eqs. (11) to represent the perigee precession with respect to the Sun direction

$$
{\frac{d\left(\bar{\omega}-\bar{\lambda}_{\mathrm{Sun}}\right)}{d t}}_{\mathrm{SRP}}=\frac{\Delta \omega_{\mathrm{SRP}, 2 \pi}}{2 \pi} \sqrt{\frac{\mu}{a^{3}}}-n_{\text {Earth-Sun }}
$$

where $n_{\text {Earth-Sun }}=\sqrt{\frac{\mu_{\text {Sun }}}{A U^{3}}}$ is the rotational rate of the Earth around the Sun equal to $0.9856 \frac{\mathrm{deg}}{\text { day }}$.

\section{B. Secular variation of Keplerian element due to atmospheric drag}

If the spacecraft orbits below a certain altitude from the Earth's surface (around $1000 \mathrm{~km}$ ), its motion is also influenced by atmospheric drag acceleration

$$
\mathbf{a}_{\text {Drag }}=-\frac{1}{2} \frac{c_{D} A_{\text {Drag }}}{m} \rho v_{r e l}^{2} \hat{\mathbf{v}}_{r e l}
$$

where $c_{D}$ is the drag coefficient, $A_{\text {drag }}$ is the effective cross-sectional area of the spacecraft and $m$ its mass, $v_{r e l}$ is the velocity relative to the rotating atmosphere and $\hat{\mathbf{v}}_{r e l}$ the corresponding versor. The secular disturbing effect on the orbit due to atmospheric drag can be modeled analogously to the case of solar radiation pressure. Starting from Gauss' equations written as function of the eccentric anomaly, King-Hele [28] derived equations which express the secular perturbation on the orbital elements due to atmospheric drag. These equations are based on the assumption of a time-independent, spherically-symmetric atmosphere with a density that varies exponentially with altitude $h$, according to:

$$
\rho=\rho_{0} \exp \left[-\frac{h-h_{0}}{H}\right]
$$

where $\rho_{0}$ is the reference density at the reference altitude $h_{0}$ and $H$ is the scale height, whose values are taken from tables [29]. If we neglect atmospheric rotation (i.e., the angular velocity of the Earth $\omega_{\text {Earth }}$ is zero), the variation of $\Delta i_{\text {Drag, } 2 \pi}, \Delta \Omega_{\text {Drag, } 2 \pi}$, and $\Delta \omega_{\text {Drag, } 2 \pi}$ due to drag is zero, while the change of the in-plane orbital elements over a single revolution is given by [22]: 


$$
\begin{aligned}
& \Delta e_{\operatorname{Drag}, 2 \pi}=-2 \pi \delta a \rho_{p} \exp [-c]\left\{I_{1}+\frac{e}{2}\left(I_{0}+I_{2}\right)-\frac{e^{2}}{8}\left(5 I_{1}-I_{3}\right)-\frac{e^{3}}{16}\left(5 I_{0}+4 I_{2}-I_{4}\right)+O\left(e^{4}\right)\right\} \\
& \Delta h_{p \operatorname{Drag}, 2 \pi}=-2 \pi \delta a^{2} \rho_{p} \exp [-c]\left\{\left(I_{0}-I_{1}\right)-\frac{e}{2}\left(3 I_{0}-4 I_{1}+I_{2}\right)+\frac{e^{2}}{8}\left(6 I_{0}-11 I_{1}+6 I_{2}-I_{3}\right)+\right. \\
& \left.-\frac{e^{3}}{16}\left(7 I_{0}-12 I_{1}+8 I_{2}-4 I_{3}+I_{4}\right)+O\left(e^{4}\right)\right\} \\
& \Delta a_{\text {Drag, } 2 \pi}=\frac{\Delta h_{p \text { Drag, } 2 \pi}+a \cdot \Delta e_{\text {Drag, } 2 \pi}}{1-e} \\
& \Delta \omega_{\text {Drag, } 2 \pi}=0
\end{aligned}
$$

where $\rho_{p}$ is the density at the orbit perigee, computed through Eq. (13), $\delta=\frac{Q A_{\mathrm{drag}} c_{D}}{m}$ (note that the drag coefficient $c_{D}$ is considered constant), the factor $c=\frac{a e}{H}$, and $I_{k}$ are the modified Bessel functions of the first kind of order $k$ and argument $c$ [30 Cap. 9]. The assumption of a static atmosphere helps to simplify the problem since it decouples the variation of the out-of-plane orbital elements from the variation of semi-major axis, eccentricity and anomaly of the pericentre. In particular, since the change in inclination is zero, the orbit always lies in the ecliptic plane. Note that Eqs. (14) are valid up to the order of eccentricity indicated, within the range $0.01 \leq e \leq 0.8$. Blitzer provides an expression of both $\Delta a_{\text {Drag, } 2 \pi}, \Delta e_{\text {Drag, } 2 \pi}$, and $\Delta h_{p \text { Drag, } 2 \pi}$, which are interdependent [22]. It was chosen to compute $\Delta e_{\text {Drag, } 2 \pi}$ and $\Delta h_{p \text { Drag, } 2 \pi}$ and to derive $\Delta a_{\text {Drag, } 2 \pi}$ from those two expressions as shown in Eqs. (14). This choice minimizes the numerical errors when the equations giving the corresponding rate of change [see Eqs. (15) in the following] are integrated over a long duration, because smaller errors (due to the higher terms in eccentricity) are cumulated in the computation of the perigee height, which determines the value of the air density.

In Section V the validity of Eqs. (6) and Eqs. (14) is verified by comparison with the numerical integration of the dynamics in Cartesian coordinates, using the expression for the disturbing accelerations Eq. (1) and Eq. (12).

Analogously to Eqs. (11), we obtain the secular and long-period rate of change of the orbital elements by dividing Eqs. (14) by the Keplerian orbital period:

$$
\begin{aligned}
& \frac{d \bar{a}}{d t_{\text {Drag }}}=\frac{\Delta a_{\text {Drag, } 2 \pi}}{2 \pi} \sqrt{\frac{\mu}{a^{3}}} \\
& \frac{d \bar{e}}{d t_{\text {Drag }}}=\frac{\Delta e_{\text {Drag, } 2 \pi}}{2 \pi} \sqrt{\frac{\mu}{a^{3}}} \\
& \frac{d \bar{\omega}}{d t_{\text {Drag }}}=0
\end{aligned}
$$

The total secular variation of the orbital elements can be expressed as:

$$
\begin{aligned}
& \frac{d \bar{a}}{d t}=\frac{d \bar{a}}{d t}_{\mathrm{SRP}}+\frac{d \bar{a}}{d t} \text { Drag } \\
& \frac{d \bar{e}}{d t}=\frac{d \bar{e}}{d t}_{\mathrm{SRP}}+\frac{d \bar{e}}{d t}{ }_{\mathrm{Drag}} \\
& \frac{d \bar{\omega}}{d t}=\frac{d \bar{\omega}}{d t}_{\mathrm{SRP}}
\end{aligned}
$$




\section{Condition for long-lived orbits}

To study the effect on the satellite's orbit of the influence of both solar radiation pressure and atmospheric drag, the secular variation of the Keplerian elements over a single orbital revolution can be computed by adding Eqs. (6) to Eqs. (14), according to the principle of superimposition. This can be done under the assumption that the coupling between SRP and atmospheric drag is negligible to first order. This simplifying assumption will be verified through numerical integration in Section V. Therefore, we obtain:

$$
\begin{aligned}
& \Delta a_{\text {Drag }+\mathrm{SRP}, 2 \pi}=\Delta a_{\text {Drag }, 2 \pi}+\Delta a_{\mathrm{SRP}, 2 \pi} \\
& \Delta e_{\text {Drag }+\mathrm{SRP}, 2 \pi}=\Delta e_{\text {Drag, } 2 \pi}+\Delta e_{\mathrm{SRP}, 2 \pi} \\
& \Delta \omega_{\text {Drag }+\mathrm{SRP}, 2 \pi}=\Delta \omega_{\text {Drag, } 2 \pi}+\Delta \omega_{\mathrm{SRP}, 2 \pi}
\end{aligned}
$$

The search for equilibrium orbits imposes three conditions to be satisfied. The total variation of semi-major axis and eccentricity due to SPR and drag must be zero, i.e., the combined effect of the two natural perturbations must cancel. Moreover, the sun-synchronous condition imposes the requirement that the change in argument of perigee over one orbit due to SRP (recall from Eqs. (14) that $\Delta \omega_{\text {Drag, } 2 \pi}=0$ ) must be equal to the angular displacement of the Earth around the Sun (i.e., the apparent rotation of the Sun around an Earth inertial system) over one orbital period of the spacecraft, such that the net change of $\omega$ is zero.

$$
\left\{\begin{array}{l}
\Delta a_{\mathrm{SRP}, 2 \pi}+\Delta a_{\mathrm{Drag}, 2 \pi}=0 \\
\Delta e_{\mathrm{SRP}, 2 \pi}+\Delta e_{\mathrm{Drag}, 2 \pi}=0 \\
\Delta \omega_{\mathrm{SRP}, 2 \pi}=\Delta \lambda_{\mathrm{Sun}, 2 \pi}
\end{array}\right.
$$

with $\Delta \lambda_{\text {Sun, } 2 \pi}=n_{\text {Earth-Sun }} \cdot T(a), T$ being the orbital period, given by Eq. (10), which depends only on the semi-major axis. Note that the third of equations (17) in the simplified case without Earth shadow was adopted by McInnes et al. [18] and Oyama et. al [19] for determining the required characteristic acceleration of a solar sail to provide sunsynchronous apse-line precession.

Before focusing on the solution of Eqs. (17), we analyze the behavior of $\Delta a_{\mathrm{SRP}, 2 \pi}, \Delta a_{\mathrm{Drag}, 2 \pi}, \Delta e_{\mathrm{SRP}, 2 \pi}$, $\Delta e_{\mathrm{Drag}, 2 \pi}$, and $\Delta \omega_{\mathrm{SRP}, 2 \pi}$ as a function of the initial orbit conditions of the satellite (i.e., the values of the Keplerian elements $a, e$, and $\omega-\lambda_{\text {Sun }}$ at the orbit pericentre). Fig. 3 to Fig. 5 show respectively the variation of semi-major axis, eccentricity and anomaly of the pericentre over a single orbit revolution due to SRP and drag, as a function of the initial condition in eccentricity and angular displacement with respect to the Sun-direction $\omega-\lambda_{\text {Sun }}$. The characteristic area-to-mass ratio for this example is reported in Table 1 (SpaceChip 2), however the following analysis is valid for all the SpaceChips proposed in Section IV.A. Due to the asymmetry introduced by eclipses, the net change in semi-major axis due to solar radiation pressure is not zero and its sign depends on the orientation of the orbit with respect to the Sun (see Fig. 3a). Due to the presence of the Earth's shadow, the increase in energy that the spacecraft gains over the half of the orbit while moving away from the Sun is not balanced by the energy loss when moving towards the Sun. This effect is greater the more the asymmetry of the orbit geometry with respect to the Sun-line is emphasized (i.e., $\omega-\lambda_{\text {Sun }}$ far from 0 or $\pi$ ). The effect of drag, instead, is not conservative and causes a constant energy loss (see Fig. 3b). It is straightforward to see that, when the two effects are superimposed, the zero of $\Delta a_{\mathrm{SRP}, 2 \pi}+\Delta a_{\text {Drag, } 2 \pi}$ moves to values of $\omega-\lambda_{\text {Sun }}$ within the range $0<\omega-\lambda_{\text {Sun }}<\pi$ (see Fig. 3c and d). The change of eccentricity over a single orbit under the influence of solar radiation pressure is a function of $\sin \left(\omega-\lambda_{\text {Sun }}\right)$ in addition to other terms due to the presence of eclipses (see Fig. 4a). Atmospheric drag causes a constant decrease in eccentricity (see Fig. $4 b$ ), hence the superimposition of the effects, moves the zero of $\Delta e_{\mathrm{SRP}, 2 \pi}+\Delta e_{\mathrm{Drag}, 2 \pi}$ towards a range of angular displacements $\pi<\omega-\lambda_{\mathrm{Sun}}<2 \pi$ (see Fig. $4 \mathrm{c}$ and d). Finally the change in anomaly of the pericentre due to solar radiation pressure varies as $\cos \left(\omega-\lambda_{\text {Sun }}\right)$ in addition to other terms due to eclipses (see Fig. 5a) and it has to counteract the angular rotation of the Earth around the Sun over one orbital period of the spacecraft, always positive (see Fig. 5b). From Fig. 5c we can conclude that this can be verified for a 
range of angular displacements $\frac{\pi}{2}<\omega-\lambda_{\mathrm{Sun}}<\frac{3}{2} \pi$. The intersection between $\Delta \omega_{\mathrm{SRP}, 2 \pi}$ and $\Delta \lambda_{\mathrm{Sun}, 2 \pi}$ can be also seen in Fig. 6 for a fixed value of perigee height.
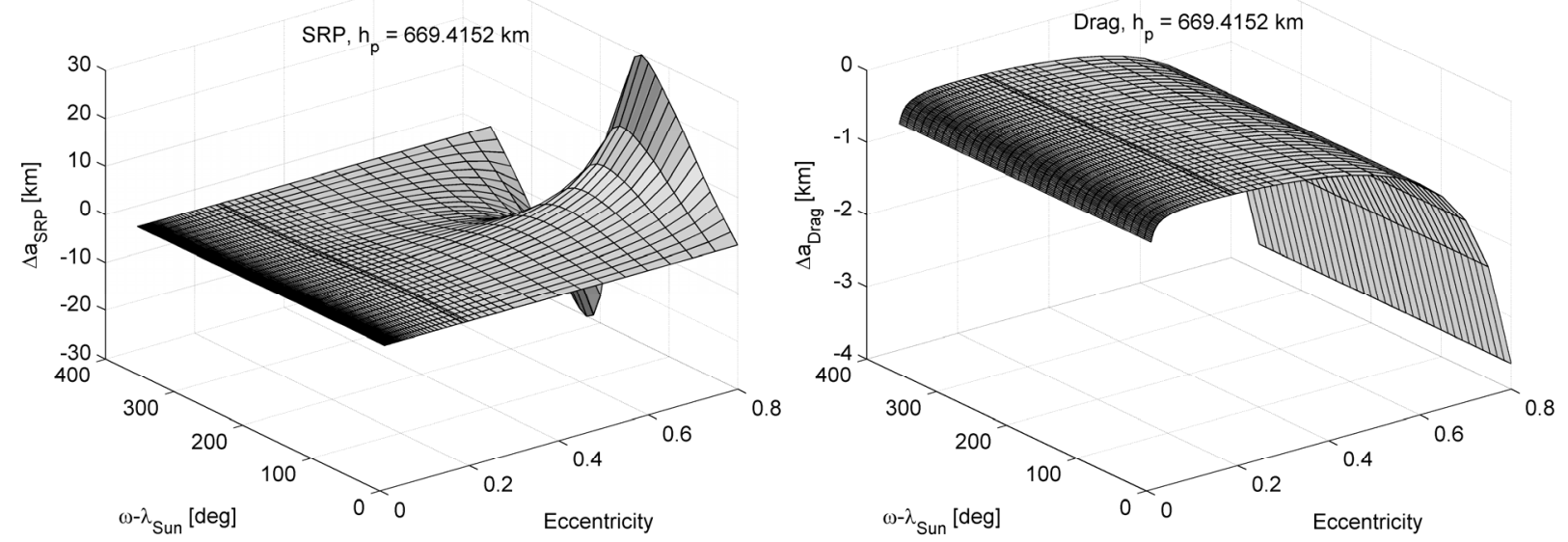

a)

b)
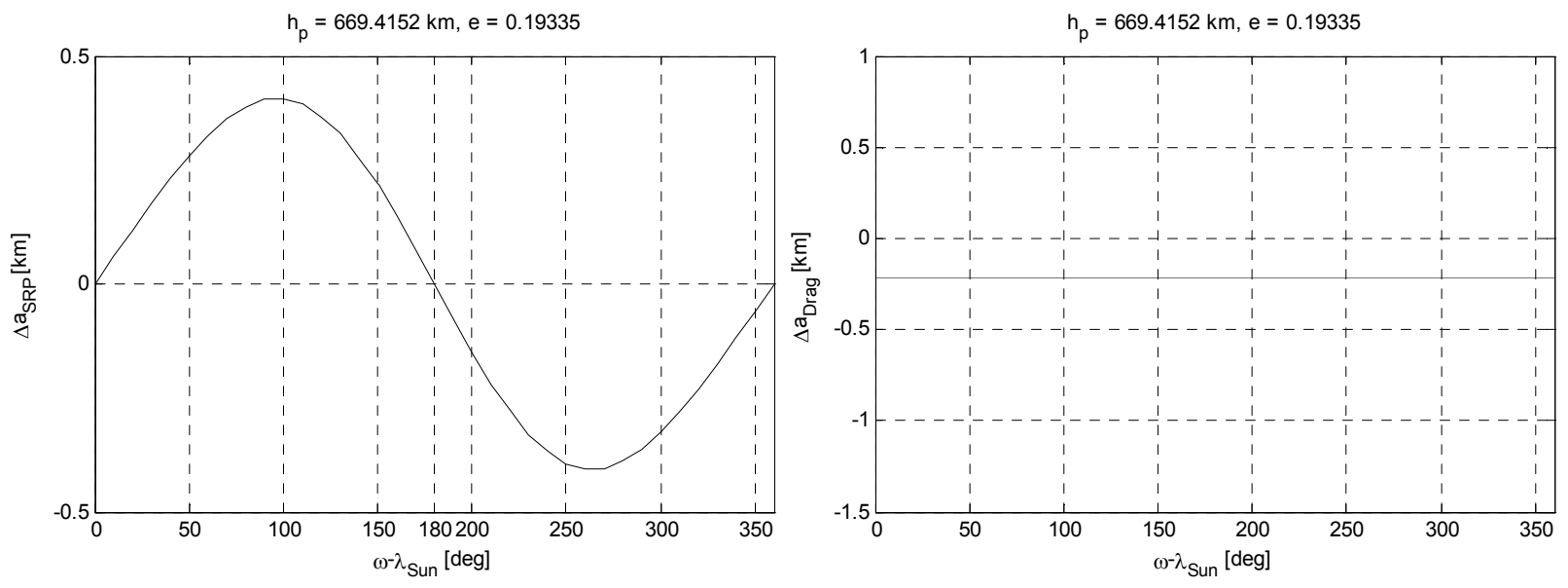

c)

d)

Fig. 3 Variation of semi-major axis over a single orbit revolution for SpaceChip 2 due to SRP and drag as a function of the initial condition in eccentricity and $\omega-\lambda_{\text {Sun }}$; a) change due to SRP, b) change due to atmospheric drag, c) evolution of $\Delta a$ due to SRP as function of $\omega$ - $\lambda_{\text {Sun, }}$ and d) evolution of $\Delta a$ due to drag as function of $\omega-\lambda_{\text {Sun }}$. 

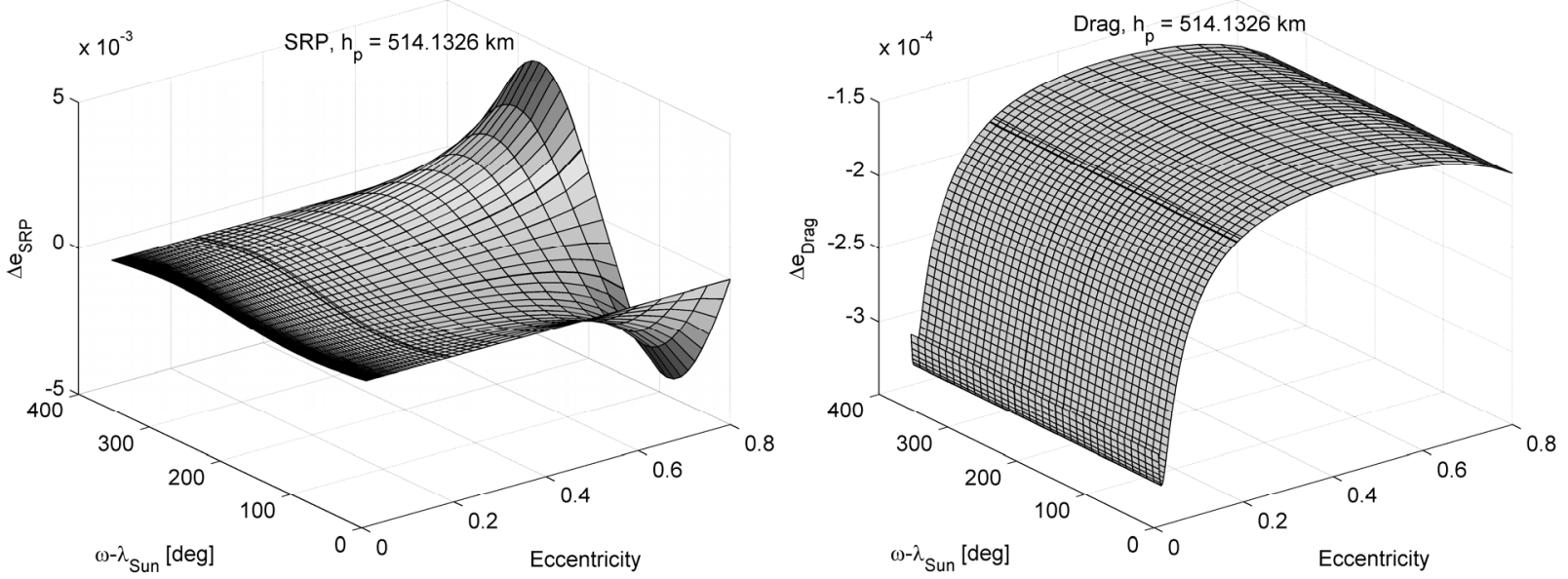

a)

b)
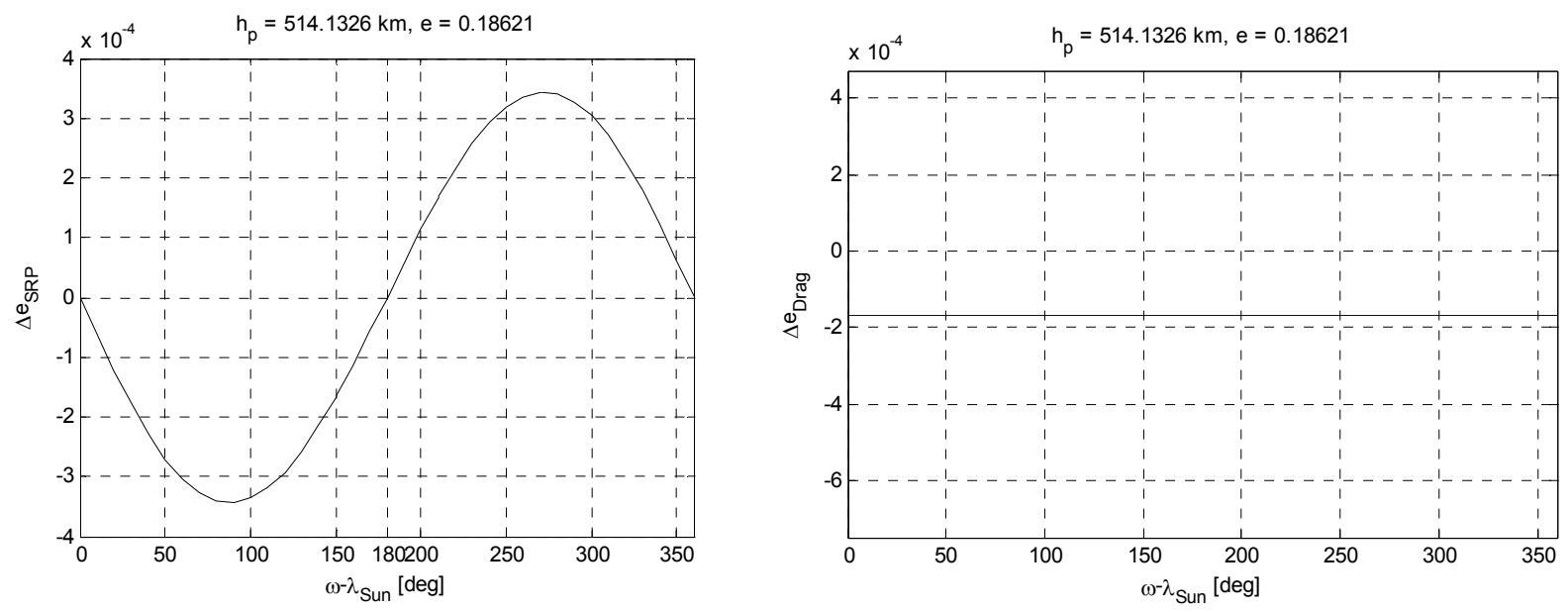

c)

d)

Fig. 4 Variation of eccentricity over a single orbit revolution for SpaceChip 2 due to SRP and drag as a function of the initial conditions in eccentricity and $\omega-\lambda_{\text {Sun }}$; a) change due to SRP, b) change due to atmospheric drag, c) evolution of $\Delta e$ due to SRP as function of $\omega$ - $\lambda_{\text {Sun }}$, and d) evolution of $\Delta e$ due to drag as function of $\omega-\lambda_{\text {Sun }}$.
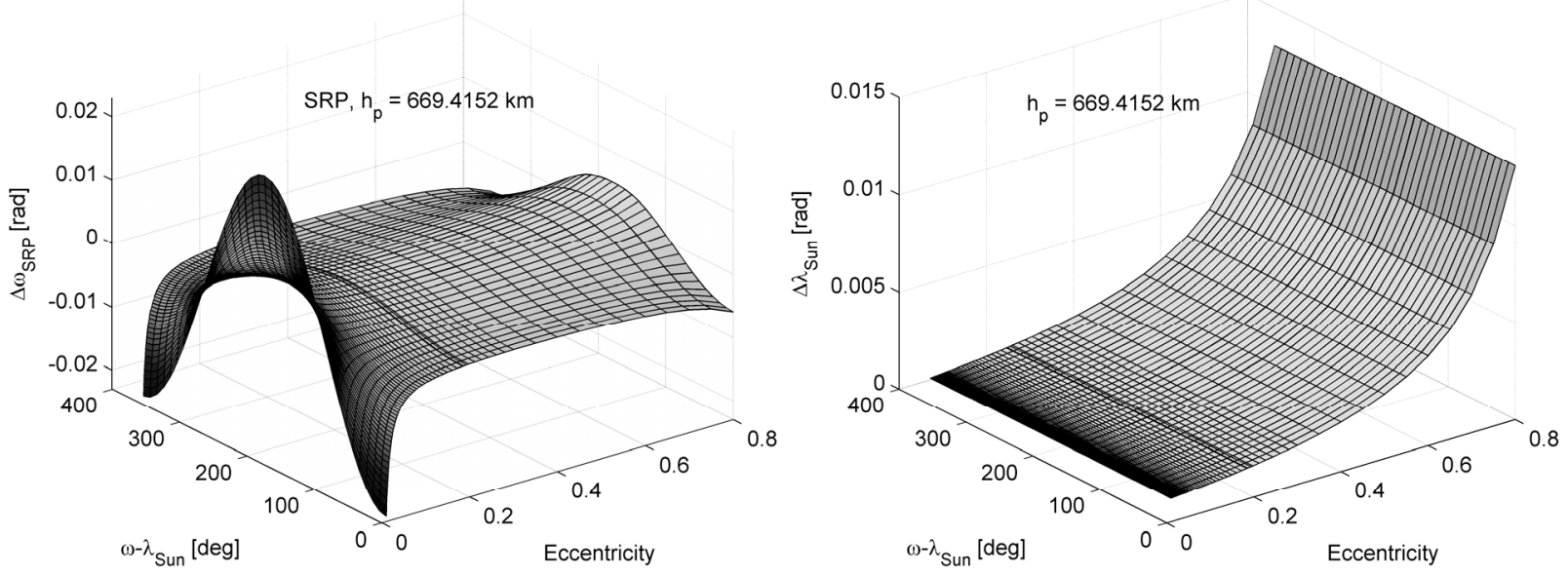

a)

b)

American Institute of Aeronautics and Astronautics 

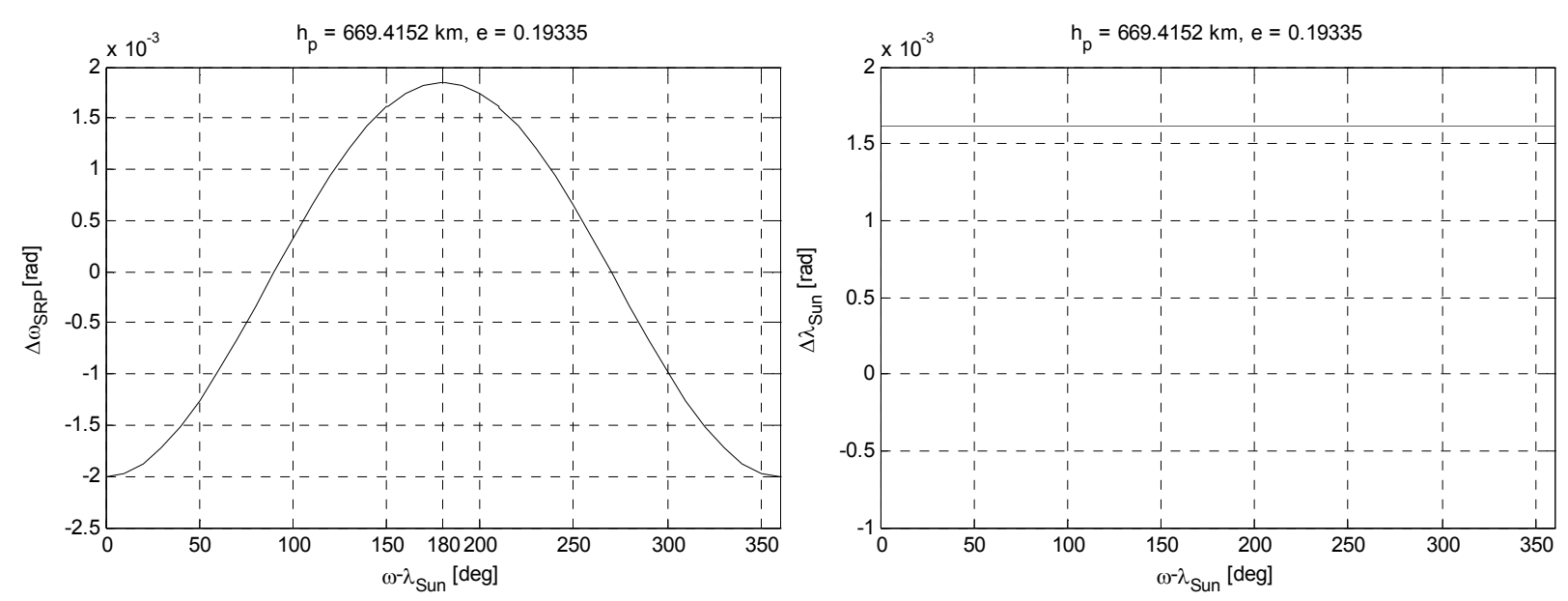

c)

d)

Fig. 5 Variation of anomaly of the pericentre due to SRP and precession of the Sun-line over a single orbit revolution for SpaceChip 2 as a function of the initial conditions in eccentricity and $\omega-\lambda_{\text {Sun }}$ a) change due to SRP, b) Sun-line precession, c) evolution of $\Delta \omega$ due to SRP as function of $\omega-\lambda_{\text {Sun }}$, and d) evolution of $\Delta \lambda_{\text {Sun }}$ as function of $\omega-\lambda_{\text {Sun }}$.

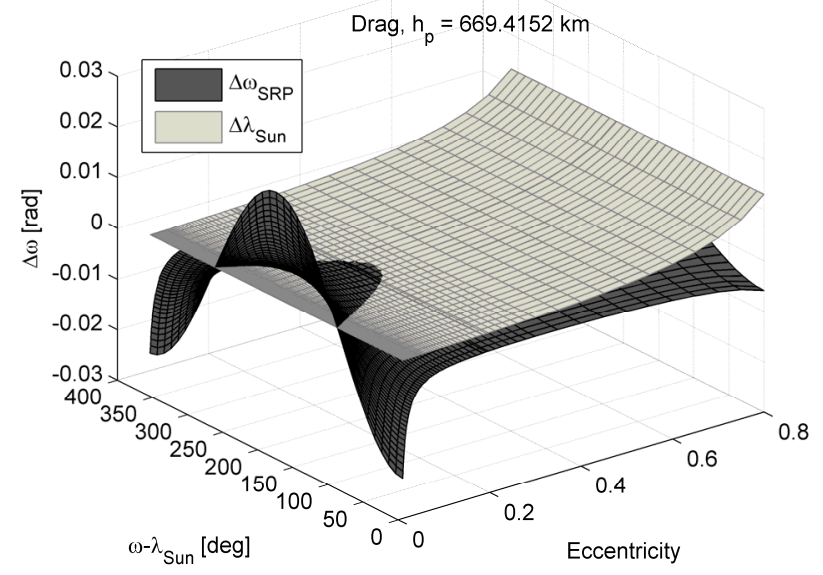

Fig. 6 Intersection between the variation of anomaly of the pericentre due to SRP and precession of the Sunline over a single orbit revolution for SpaceChip 2 as a function of the initial condition in eccentricity and $\omega$ $\lambda_{\text {Sun. }}$

To summarize, from the analysis of Fig. 3 to Fig. 5 it is possible to draw conclusions on each of the equations in system Eq. (17), and to limit the domain of angular displacements where solutions can be found; the first of Eqs. (17) can be zero within the range $0 \leq \omega-\lambda_{\text {Sun }} \leq \pi$ and the total variation of eccentricity [second of Eqs. (17)] can be zero for $\pi \leq \omega-\lambda_{\text {Sun }} \leq 2 \pi$ (in both the equations, the limit values are for the case of SRP only). The Sunsynchronous condition $\Delta \omega_{\mathrm{SRP}, 2 \pi}=\Delta \lambda_{\mathrm{Sun}, 2 \pi}$ can be solved for $\frac{\pi}{2} \leq \omega-\lambda_{\mathrm{Sun}} \leq \frac{3}{2} \pi$ (the limit values are for a circular orbit as can be seen in Fig. 6). Therefore, we can confirm that the system Eq. (17) cannot be satisfied if both solar radiation pressure and atmospheric drag are present. If the effect of drag is negligible, equilibrium orbits can be identified under the effect of solar radiation pressure for $\omega-\lambda_{\text {Sun }}=\pi$; this concept was exploited by McInnes et al. [18] and Oyama et. al [19]. When solar radiation pressure and atmospheric drag both have a non-negligible effect on the spacecraft orbit, even if a complete equilibrium is not possible, it is useful to study partial equilibrium solutions: 


$$
\begin{aligned}
& \left\{\begin{array}{l}
\Delta a_{\mathrm{SRP}, 2 \pi}+\Delta a_{\mathrm{Drag}, 2 \pi}=0 \\
\Delta \omega_{\mathrm{SRP}, 2 \pi}=\Delta \lambda_{\mathrm{Sun}, 2 \pi}
\end{array}\right. \\
& \left\{\begin{array}{l}
\Delta e_{\mathrm{SRP}, 2 \pi}+\Delta e_{\mathrm{Drag}, 2 \pi}=0 \\
\Delta \omega_{\mathrm{SRP}, 2 \pi}=\Delta \lambda_{\mathrm{Sun}, 2 \pi}
\end{array}\right.
\end{aligned}
$$

in which the Sun-synchronous condition is satisfied and only one variation, either semi-major axis or eccentricity is zero. These solutions are visualized in Fig. 7 for SpaceChip 2 whose characteristics are reported in Table 1. Fig. 7a shows an example for which the total variation of semi-major axis (dashed line) due to SRP and drag is zero, and the Sun-synchronous condition is satisfied $\left(\Delta \omega_{\mathrm{SRP}, 2 \pi}\right.$ is represented by the continuous black line, $\Delta \lambda_{\mathrm{Sun}, 2 \pi}$ is represented by the continuous black bold line). Fig. 7b reports a solution of the system Eq. (19) for which the total variation of eccentricity (dotted line) due to SRP and drag is zero, and the Sun-synchronous condition is satisfied. The meaning of these partial equilibrium solutions of the systems Eq. (18) and Eq. (19) will be explained later in Section V.
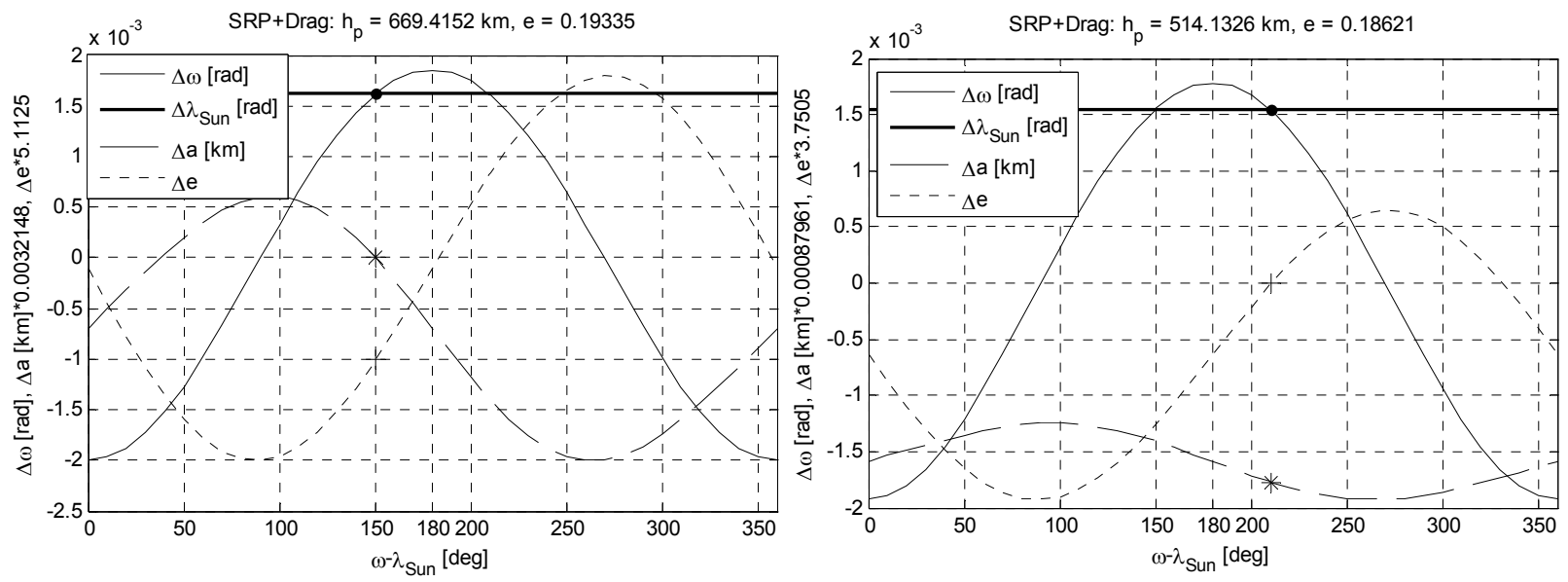

a)

b)

Fig. 7 Variation of semi-major axis, eccentricity, anomaly of the pericentre over a single orbit revolution for SpaceChip 2 due to SRP and drag as a function of the initial condition in $\omega-\lambda_{\text {Sun. }}$ a) Condition for $\Delta a=0$ with the Sun-synchronous condition satisfied, and b) Condition for $\Delta e=0$ with the Sun-synchronous condition satisfied.

When only solar radiation pressure, without the Earth's shadow, is considered, the solution of system Eq. (17) can be determined analytically [18-19]. In the more complex case we consider, an analytical closed-form solution of Eqs. (17)-(19) is not possible; therefore, the problem is solved numerically. In this paper a global multi-start approach is adopted. A local algorithm is started from several points randomly distributed over the entire domain of semi-major axis, eccentricity and arguments of angular displacement $\omega-\lambda_{\text {Sun }}$. For the results included in this paper we set $3.2 \times 10^{5}$ sampling points for the solution of each of the systems Eq. (17)-(19). Starting from each point on the mesh, a local minimization is performed numerically, through a subspace trust-region method, based on the interior-reflective Newton method [31-32]. The objective function used for the minimization is:

$$
\min _{\left\{a, e, \omega-\lambda_{\text {Sun }}\right\}}\left[w_{a}\left|\Delta a_{\text {Drag, } 2 \pi}+\Delta a_{\mathrm{SRP}, 2 \pi}\right|+w_{e}\left|\Delta e_{\text {Drag }, 2 \pi}+\Delta e_{\mathrm{SRP}, 2 \pi}\right|+w_{\omega}\left|\Delta \omega_{\text {Drag }, 2 \pi}+\Delta \omega_{\mathrm{SRP}, 2 \pi}-\Delta \lambda_{\mathrm{Sun}, 2 \pi}\right|\right]
$$

where $w_{a}, w_{e}, w_{\omega}$ are weight parameters introduced to treat this multi-objective minimization problem (the three equations of Eqs. (17) must be solved) as a single-objective minimization. Depending on the value of the weight parameter, the multi-start algorithm can identify the solution set of system Eq. (17), Eq. (18), or Eq. (19). The ratio between $w_{a}$ and $w_{\omega}$, and $w_{e}$ and $w_{\omega}$ were chosen such that, for any solution found the Sun-synchronous apse-line 
condition is always satisfied. Gauss' equations in form Eq. (3) are singular for $e=0$ and are valid for elliptic orbits; hence we restricted the eccentricity to be within the range $0.01 \leq e \leq 0.8$ which is of practical interest.

\section{Results for partial equilibrium orbits}

In the following section, the numerical results of the solution of systems Eq. (17), Eq. (18) and Eq. (19) will be shown, in the convenient representation of the phase-space.

\section{A. Spacecraft and perturbation model}

We consider a silicon microchip with density $2.3 \mathrm{~g} / \mathrm{cm}^{3}\left(=2300 \mathrm{~kg} / \mathrm{m}^{3}\right)$, and dimensions reported in Table 1 . Three different cases are analyzed, corresponding to increasing values of area-to-mass ratio. Table 1 reports the dimension of a microchip, $1 \mathrm{~cm}$ square, with different thickness values $l_{2}$, and for comparison, the radius $l$ of a sphere with an equivalent area-to-mass ratio. The sphere shape is usually adopted for studies on interplanetary dust. Since the SpaceChip density is assumed uniform, the characteristic length is represented by the chip's thickness:

$$
\begin{array}{rlrl}
A_{\text {chip }} & =l_{1}^{2} & \frac{A}{m}=\frac{1}{l_{2} \cdot \rho_{\text {silicon }}} \\
m_{\text {chip }} & =l_{1}^{2} \cdot l_{2} \cdot \rho_{\text {silicon }} & \\
A_{\text {sphere }} & =\pi l^{2} & & \frac{A}{m}=\frac{1}{\frac{4}{3} l \cdot \rho_{\text {silicon }}} \\
m_{\text {sphere }} & =\frac{4}{3} \pi l^{3} \cdot \rho_{\text {silicon }}
\end{array}
$$

where $A$ is the cross-section area. The size of SpaceChip 1 (see Table 1) was taken from the design by Atchison and Peck [4], hence it represents a near-term device. SpaceChip 2 and 3 represent scenarios with lower technology readiness levels; however they were selected to show the sensitivity of the area-to-mass-ratio on the conditions for long-lived orbits discussed later.

In this paper we consider that the average effective cross-section $A_{\odot}$ exposed to the Sun is always equal to the cross-section area of the spacecraft $A$. This implies that the spacecraft has a spherical shape or its attitude is kept fixed with respect to the Sun-line. A passive Sun-pointing attitude control was proposed for millimeter-scale solar sails, based on faceted surfaces to stabilize the Sun-pointing plate [4]. Alternately, electro-chromic elements with variable reflectance can be layered at the sides of the chip to be exploited as steering device, as tested on the IKAROS mission [17]. Moreover a reflectivity coefficient $c_{R}=1.8$ is assumed (note that for a black body $c_{R}=1$, for a flat mirror perpendicular to the light direction $c_{R}=2$ ).

For the model of atmospheric drag, a drag coefficient $c_{D}=2.1$ is chosen $\left(c_{D}=2.2\right.$ is usually used for a flat plate model, $c_{D}=2.0$ to 2.1 for spherical particles), and the cross-sectional area $A_{\text {drag }}$ is considered constant and equal to the cross-section area of the spacecraft. The reference values for the computation of the air density in Eq. (13) where taken equal as [29]:

$$
\begin{aligned}
& h_{0}=600 \mathrm{~km} \\
& \rho_{0}=1.454 \times 10^{-13} \mathrm{~kg} / \mathrm{m}^{3} \\
& H=71.835 \mathrm{~km}
\end{aligned}
$$

since the region where solar radiation pressure and atmospheric drag are known to have comparable effect is around 400-800 km. A more accurate value of the density would be obtained by selecting the reference values $h_{0}, \rho_{0}$ and $H$ closest to the range of orbit altitudes considered.

Table 1 contains also the characteristic acceleration due to solar radiation pressure computed through Eq. (2). 
Table 1 SpaceChip characteristics.

\begin{tabular}{lllll}
\hline \hline & $\begin{array}{c}\text { Chip dimensions } \\
{[\mathrm{mm}]}\end{array}$ & $\begin{array}{c}\text { Sphere dimensions } \\
l[\mathrm{~mm}]\end{array}$ & $\mathrm{A} / \mathrm{m}\left[\mathrm{m}^{2} / \mathrm{kg}\right]$ & $a_{\mathrm{SRP}}\left[\mathrm{mm} / \mathrm{s}^{2}\right]$ \\
\hline SpaceChip 1 & $\begin{array}{l}l_{l}=10 \\
l_{2}=0.0250\end{array}$ & 0.0187 & 17.3913 & 0.1427 \\
\hline SpaceChip 2 & $\begin{array}{l}l_{l}=10 \\
l_{2}=0.0133\end{array}$ & 0.01 & 32.6087 & 0.2676 \\
\hline SpaceChip 3 & $\begin{array}{l}l_{1}=10 \\
l_{2}=0.00796\end{array}$ & 0.00597 & 54.6364 & 0.4484 \\
\hline \hline
\end{tabular}

\section{B. Analysis of long-lived orbits}

The solution of the systems Eqs. (17)-(19) can be represented in the phase space $\left\{e \omega-\lambda_{\text {Sun }} h_{p}\right\}$ or equivalently $\left\{\begin{array}{lll}e & \omega-\lambda_{\text {Sun }} & a\end{array}\right\}$, as depicted in Fig. 8 for missions employing SpaceChip 1. The graph is represented in three dimensions (Fig. 8d) and three two-dimension views (Fig. 8a-c). For an eccentricity higher than approximately 0.115 and perigee height above $900 \mathrm{~km}$, a set of solutions for system Eq. (17) exists with the condition $\omega-\lambda_{\text {Sun }}=\pi$. With these initial conditions the orbit perigee is along the Sun-Earth direction, facing the Sun. This position is a stable condition for the variation of $\omega-\lambda_{\text {sun }}$ and the change in eccentricity and semi-major axis over one orbit cancels, as the effect or SRP is symmetric in this configuration, and the drag is negligible (over one orbit). Considering the branch of the graph with $\omega-\lambda_{\text {Sun }}=\pi$, the greater the perigee height and the eccentricity decrease, the greater the effect of drag becomes up to a certain point at which it cannot be neglected. Beyond this point, as expected from the analysis in section III, no global equilibrium solutions [Eq. (17)] can be found. In this region of the domain (for perigee heights below $800 \mathrm{~km}$ approximately), atmospheric drag and SRP have comparable effects. Still a set of solutions exists for system Eq. (18) (i.e., $\Delta a_{2 \pi}=0$ and sun-synchronous condition satisfied) and Eq. (19) (i.e., $\Delta e_{2 \pi}=0$ and sun-synchronous condition satisfied), represented respectively by the gray colored branch and the black colored branch of the graph. Note that each point in Fig. 8 corresponds to an initial condition for an Earth-centered orbit. Fig. 8 is an example of bifurcation, a solution for global equilibrium orbits [system Eq. (17)] can be found for eccentricities and perigee heights higher than a certain value $\left\{e^{*} h_{p}^{*}\right\}$. Below, the equilibrium disappears and the only possible solution degrades to system Eq. (18) or Eq. (19) (only two out of the three equations of system Eq. (17) can be satisfied).
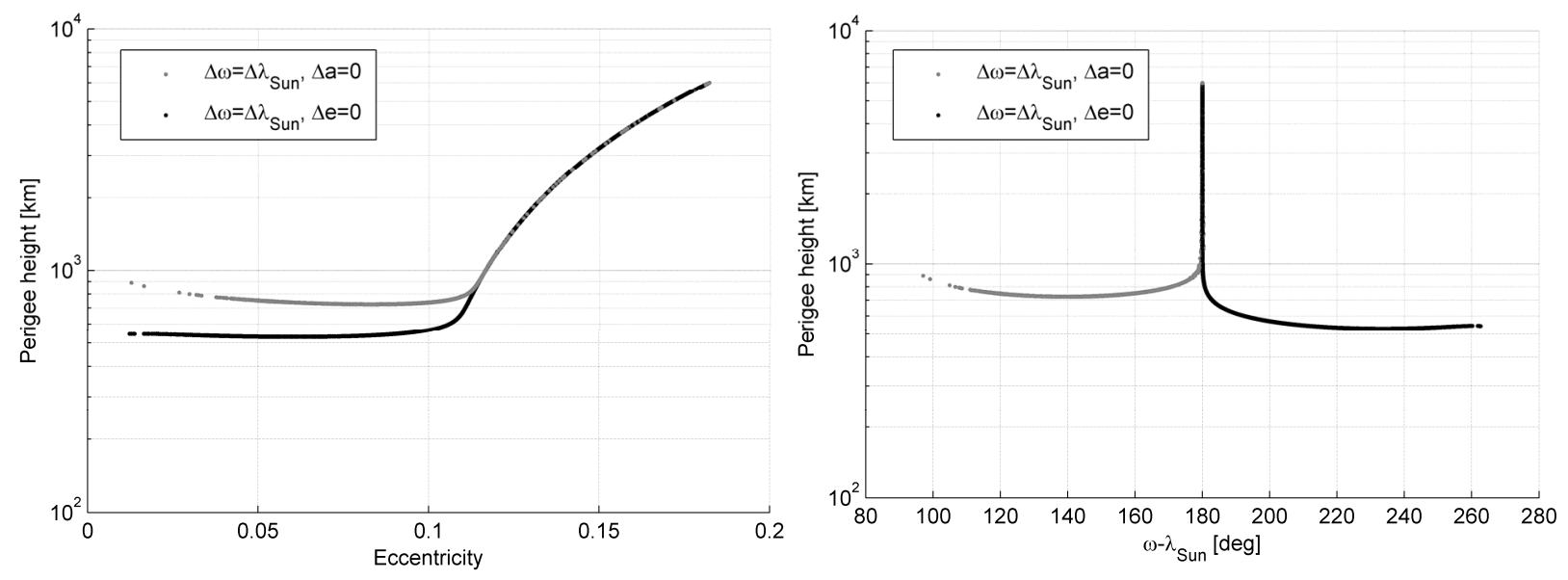

a)

b) 

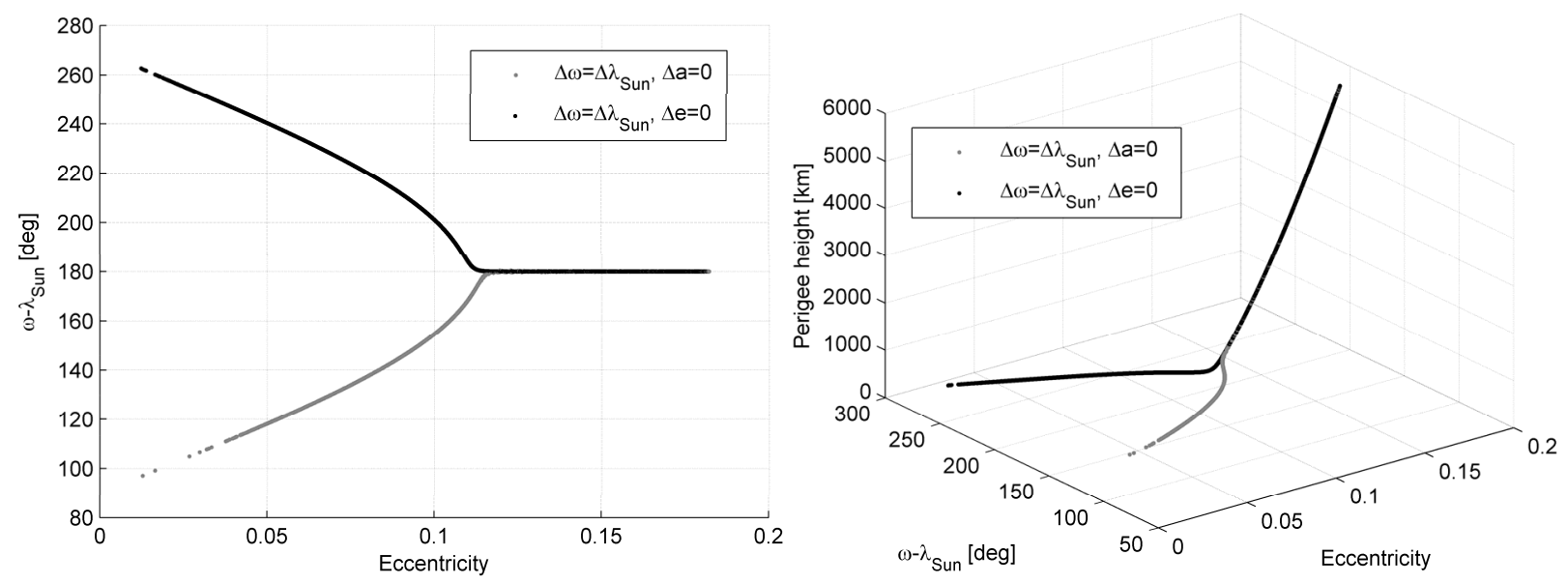

c)

d)

Fig. 8 Long-lived orbits conditions for SpaceChip 1 missions.

As expected the lines in Fig. 8 represent the intersections between the surfaces solutions of system Eq. (17) as shown in Fig. 9. The dark gray surface is the solution of the sun-synchronous condition $\Delta \omega_{\mathrm{SRP}, 2 \pi}=\Delta \lambda_{\mathrm{Sun}, 2 \pi}$, the light gray surface on the domain $\pi \leq \omega-\lambda_{\text {Sun }} \leq 2 \pi$ contains the initial conditions for which the variation of the eccentricity due to SRP and drag balances (i.e., $\Delta e_{\mathrm{SRP}, 2 \pi}+\Delta e_{\mathrm{Drag}, 2 \pi}=0$ ) and the cyan surface on the domain $0 \leq \omega-\lambda_{\text {Sun }} \leq \pi$ represents the solution of $\Delta a_{\mathrm{SRP}, 2 \pi}+\Delta a_{\mathrm{Drag}, 2 \pi}=0$. In correspondence with the condition $\omega-\lambda_{\text {Sun }}=\pi$, above a certain value of the perigee altitude, the surfaces $\Delta e_{\mathrm{SRP}, 2 \pi}+\Delta e_{\mathrm{Drag}, 2 \pi}=0$ and $\Delta a_{\mathrm{SRP}, 2 \pi}+\Delta a_{\mathrm{Drag}, 2 \pi}=0$ intersect. Both the perturbing acceleration due to solar radiation and atmospheric drag are directly proportional to the area-to-mass ratio of the spacecraft [see Eq. (2) and Eq. (12)], hence we can expect that the surfaces $\Delta a_{\mathrm{SRP}, 2 \pi}+\Delta a_{\mathrm{Drag}, 2 \pi}=0$ and $\Delta e_{\mathrm{SRP}, 2 \pi}+\Delta e_{\mathrm{Drag}, 2 \pi}=0$ remain unchanged for any area-to-mass ratio. Instead, only the surface $\Delta \omega_{\mathrm{SRP}}-\Delta \lambda_{\mathrm{Sun}, 2 \pi}=0$ changes.

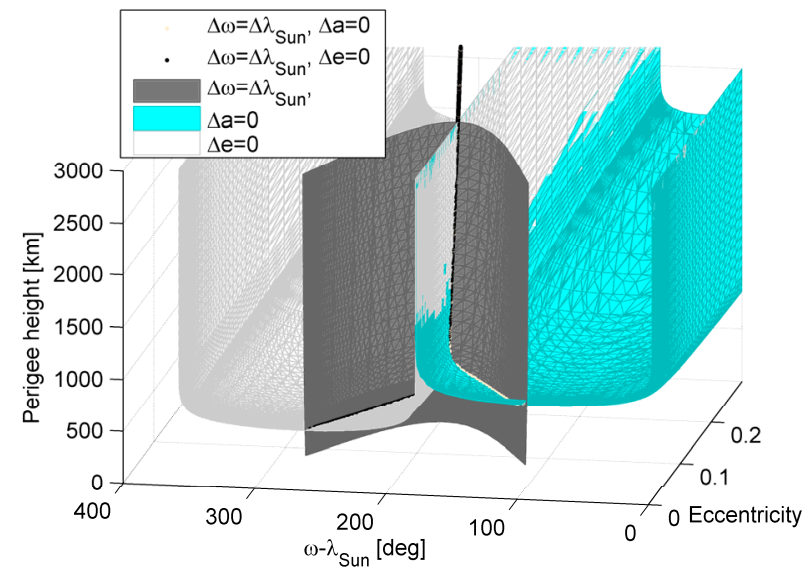

a)

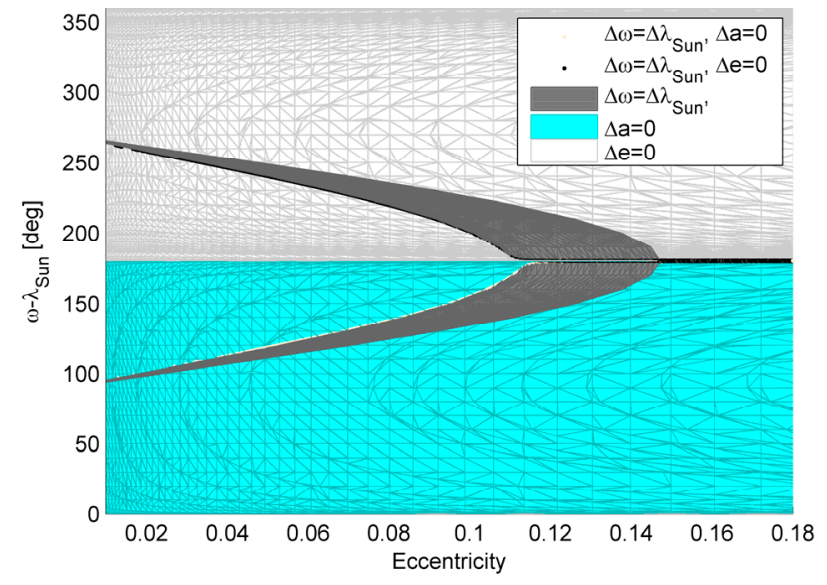

b)

Fig. 9 Surfaces representing each of the equations of system Eq. (17) for SpaceChip 1 missions. a) 3D view, and b) 2D view in eccentricity $-\omega-\lambda_{\text {Sun }}$. 
In Fig. 10 the solutions for different SpaceChip designs are shown to appreciate the sensitivity of the solution to the spacecraft area-to-mass ratio. In particular, when the parameter $\frac{A}{m}$ increases, the amplitude of $\Delta \omega_{\mathrm{SRP}, 2 \pi}$, represented in Fig. 5a, increases, but $\Delta \lambda_{\text {Sun }}$ is unchanged since it does not depend on the spacecraft characteristics; hence the intersection surface, shown in Fig. 9, is displaced for increasing values of eccentricity, as can be seen in Fig. 10.
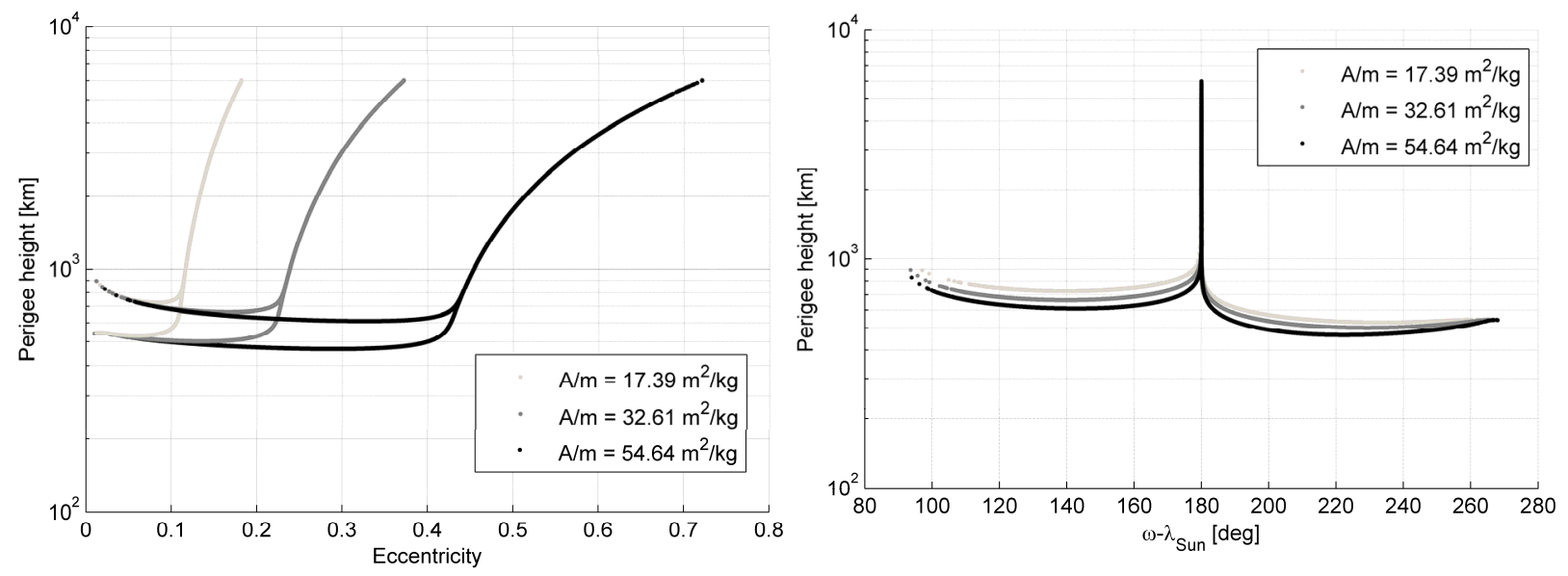

a)

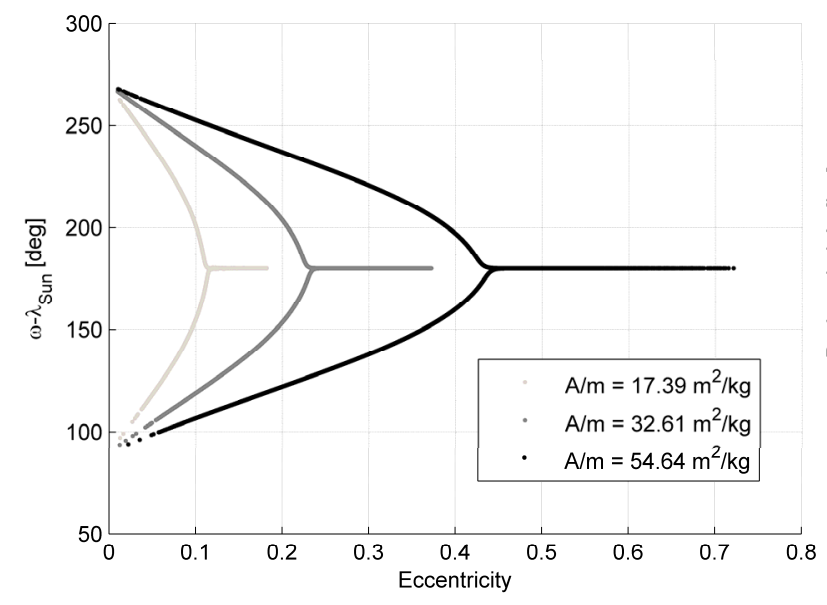

b)

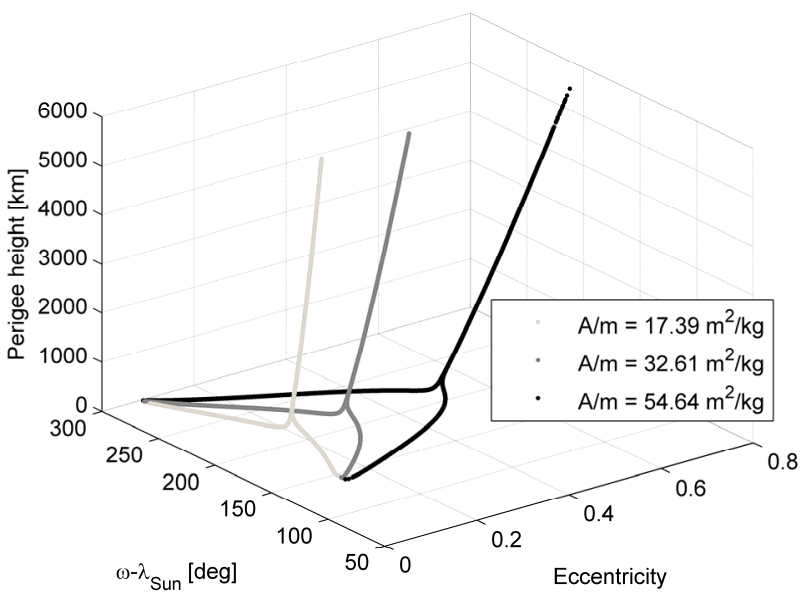

d) 

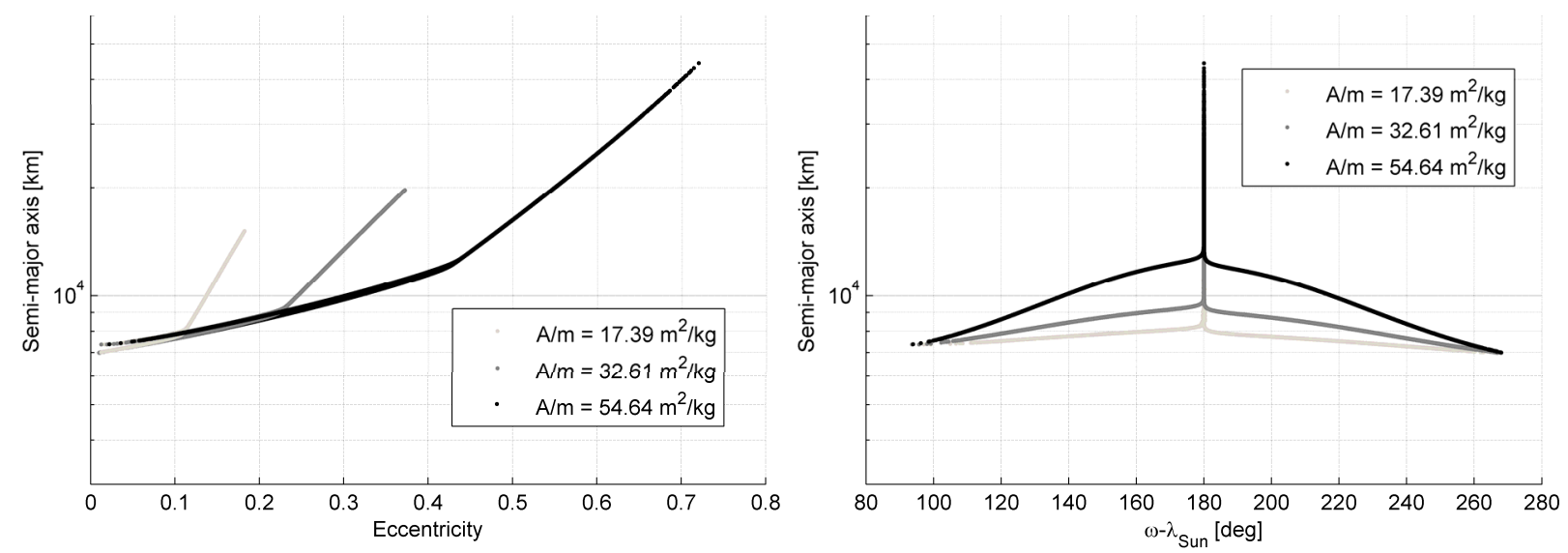

e)

f)

\section{Fig. 10 Long-lived orbits condition for SpaceChip missions.}

In the following sections we will refer to Fig. 8, and Fig. 10 as long-lived orbits surface and we will indicate the Keplerian vectors of initial conditions (i.e., each point on the long-lived orbits surface) with the symbol kep ${ }_{L L}$.

\section{Long-term orbit evolution}

In this section we analyze the long-term evolution of orbits whose initial conditions are represented by the set of points in Fig. 8 and Fig. 10. The long-term evolution is predicted by integrating Eqs. (16) and using a stopping condition for the integration:

$$
C_{\text {forw }}:\left\{h_{p} \leq 250 \mathrm{~km} \vee e \leq 0.01 \vee e \geq 0.8\right\}
$$

that is when the orbit perigee drops under a set limit of $250 \mathrm{~km}$ or the eccentricity exceeds the lower or upper bounds of 0.01 and 0.8 , which is our domain of interest. The first condition of Eqs. (20) is set because below a certain perigee altitude the model used for predicting the effect of atmospheric drag is not valid, since $H$ changes rapidly with height, and the orbital changes are no longer small enough for their squares to be ignored [28]. Anyway, the lifetime once the spacecraft transits below that altitude becomes so short that the mission is over. Later in this section, it will be useful to propagate the initial conditions backwards in time, to find the foregoing behavior of the spacecraft. In this case the stopping condition for the integration is:

$$
C_{\text {back }}:\left\{h_{p} \leq 250 \mathrm{~km} \vee h_{p} \geq h_{p, \text { up }} \vee e \leq 0.01 \vee e \geq 0.8\right\}
$$

where also an upper limit on the perigee altitude $h_{p \text {, up }}$ is introduced. The numerical integration of Eqs. (16) is performed through an adaptive step-size Runge-Kutta-Fehlberg integration scheme integrator with a six stage pair of approximation of the fourth and fifth order [33], with absolute and relative tolerance of $2.5 \times 10^{-14}$.

For a better understanding of the long-term behavior, it is useful to distinguish two different parts of the longlived orbits condition graph. We indicate with $e^{*}$ the value of the eccentricity at which the bifurcation takes place; note that the exact value of $e^{*}$ depends on the tolerances set for the solution of system Eq. (17), which determine when the solution for a stable orbit (i.e., three equations of system Eq. (17) satisfied) does not exist anymore. In that case we can still find partial equilibrium solutions represented by systems Eq. (18) and Eq. (19). According to this definition we define the sections of long-lived orbits surface as:

- $e<e^{*}$ where we can identify the two branches of the solutions sets of system Eq. (18) and Eq. (19);

- $e \geq e^{*}$ where the two branches progressively merge in the solution of system Eq. (17) 


\section{A. Region $e<e^{*}$}

We now focus on the first region of the solution space, in particular on the branch representing the solutions of systems Eq. (18) (i.e., $\Delta a_{2 \pi}=0$ and the sun-synchronous condition satisfied). The dynamics of the SpaceChip has been propagated, starting from some vector of initial conditions belonging to this set. For each initial point, the orbit has been propagated backward in time, considering the dynamics of SRP and drag [Eqs. (16)], until one of the conditions in Eq. (21) is met. Then, the final state of the backward integration is used as the initial condition for the forward integration, until one of the conditions in Eq. (20) is met. Having the highest value of area-to-mass ratio, the effect of the perturbations is most pronounced for SpaceChip 3; hence we select this scenario for the analysis in this section. In Fig. 11 the long-term evolution subjected to SRP and drag is shown with the black line, starting from the initial state indicated with the black symbol '+', until the stopping criterion Eq. (20) is met. The behavior is similar for any vector of initial conditions in the set. The orbit perigee drifts following the apparent Sun-line rotation, starting behind the Sun and moving ahead of it while the perigee altitude rises when $0<\omega-\lambda_{\text {Sun }}<\pi$ and decreases when $\pi<\omega-\lambda_{\text {Sun }}<2 \pi$. Each line bends right in correspondence to the branch with $\Delta e_{2 \pi}=0$ and $\Delta \omega_{2 \pi}=\Delta \lambda_{\text {Sun, } 2 \pi}$, and then the spacecraft orbit evolves towards decay. In this case all the end-points represented by the black dot symbol are in correspondence with the stopping condition $h_{p}=250 \mathrm{~km}$ of Eq. (20). In Fig. 11 the orbit evolution under the effect of drag only is superimposed (gray line), starting from the same initial conditions of the SRP and drag case, identified with the black symbol ' $+{ }^{\circ}$. The orbit shrinks while the radius of the perigee tends to remain constant (see Fig. 11c). The difference between the SRP and drag and drag-only case is highlighted in Fig. 11c-d. The effect of SRP causes a significant increase in the orbit lifetime with respect to the drag-only case, as shown in Fig. 12, as a function of the eccentricity of the initial condition vector ('+' symbol in Fig. 11).

The long-term forward evolution of the orbits with initial conditions which lie on the branch representing the solutions of systems Eq. (19) (i.e., $\Delta e_{2 \pi}=0$ and sun-synchronous condition satisfied), instead, lead to a fast orbit decay, and the presence of SRP contributes to a decrease of the orbit lifetime with respect to the drag-only case (see Fig. 14). This is shown in Fig. 13, where the black line represents the SRP and drag scenario, the gray line the drag-

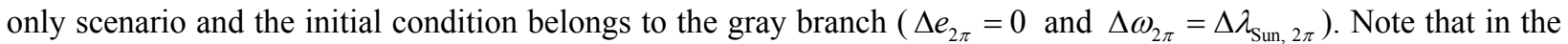
SRP and drag scenario, the orbit decay phase does not evolve at a constant perigee, as in the case of drag only. Moreover, the gray lines in Fig. $13 \mathrm{~b}$ (drag only scenario) which jump from $\omega-\lambda_{\text {Sun }}=0$ to $\omega-\lambda_{\text {Sun }}=2 \pi$ correspond to rotational motion, where $\omega-\lambda_{\text {sun }}$ continues to decrease.

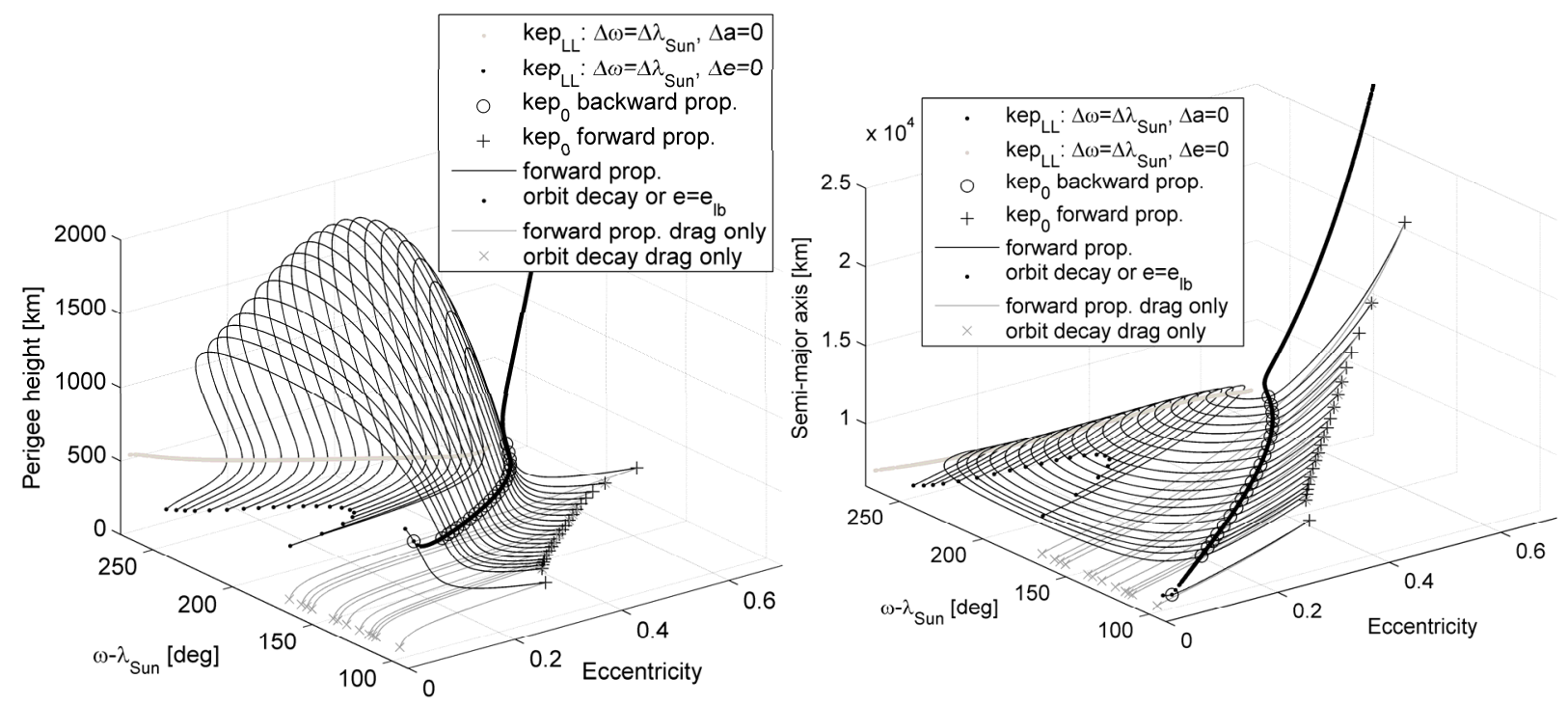

a) 

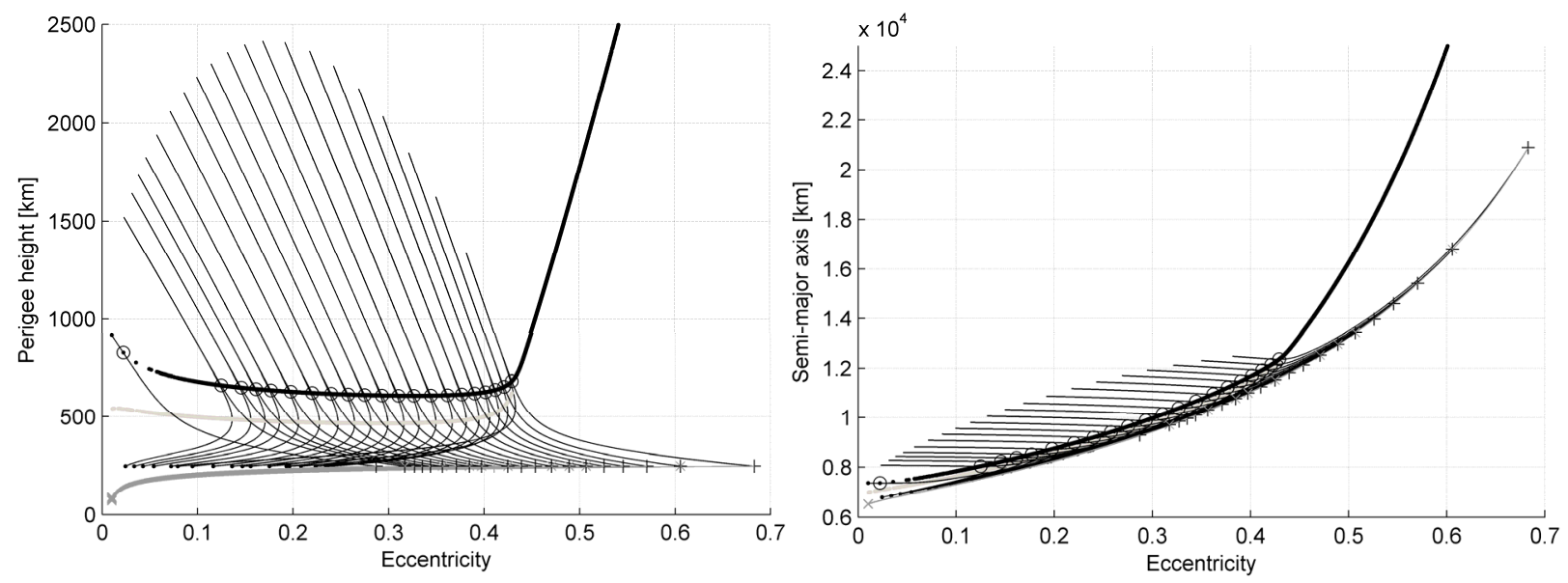

c)

d)

Fig. 11 Long-term orbit evolution for SpaceChip 3 from initial conditions with $\Delta a=0$ and $\Delta \omega=\Delta \lambda_{\text {Sun }}$. The black lines represent the SRP and drag scenario, the gray lines the drag-only scenario. a) 3D view in the phase-space with perigee height on the z-axis, b) $3 D$ view in the phase-space with semi-major axis on the z-axis, c) 2D view in perigee height-eccentricity, d) 2D view in semi-major axis-eccentricity.

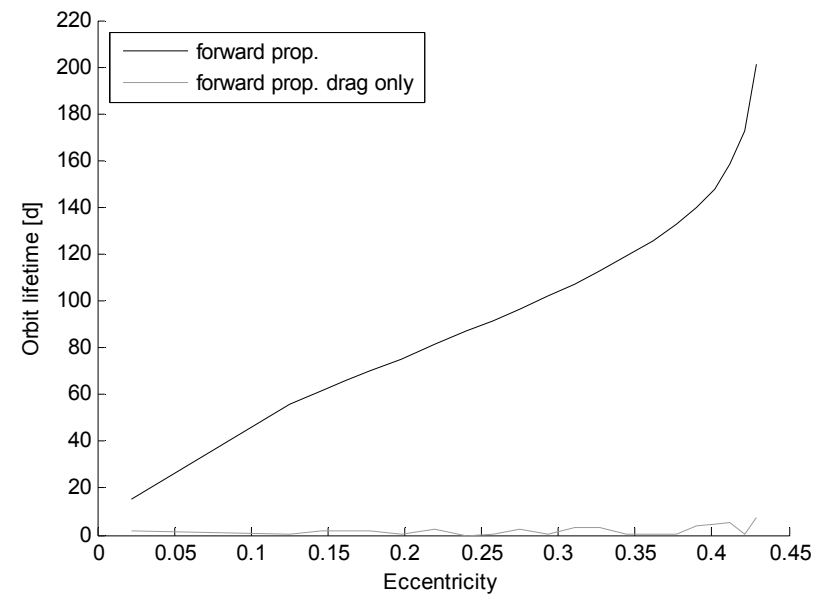

Fig. 12 SpaceChip 3 orbit lifetime for evolution from initial conditions with $\Delta a=0$ and $\Delta \omega=\Delta \lambda_{\text {Sun }}$. The black line represents the SRP and drag scenario, the gray line the drag-only scenario. 


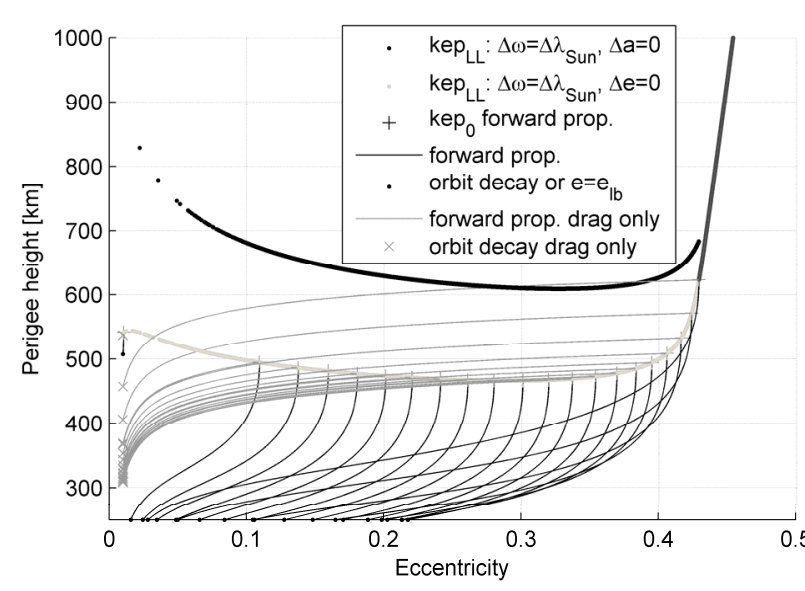

a)

Fig. 13 Long-term orbit evolution for SpaceChip 3 from initial conditions with $\Delta e=0$ and $\Delta \omega=\Delta \lambda_{\text {Sun }}$. The black lines represent the SRP and drag scenario, the gray lines the drag-only scenario.

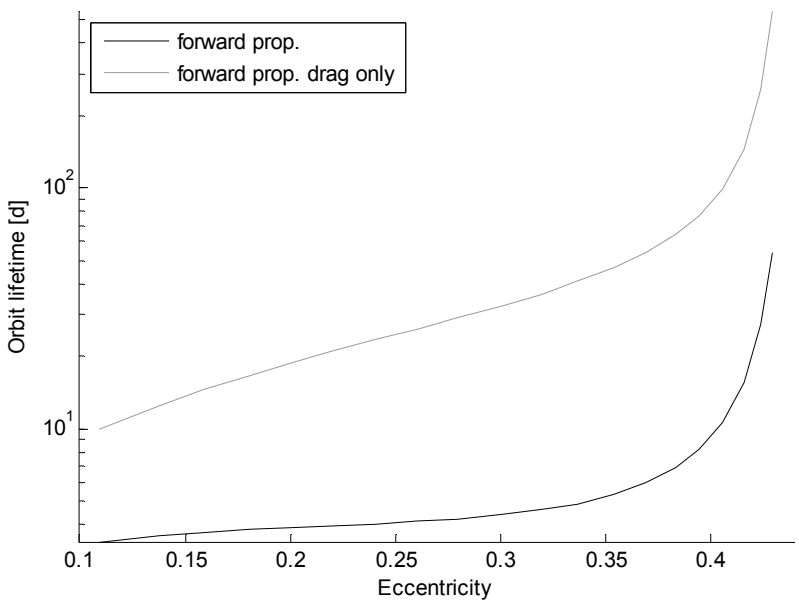

Fig. 14 SpaceChip 3 orbit lifetime for evolution from initial conditions with $\Delta e=0$ and $\Delta \omega=\Delta \lambda_{\text {Sun }}$. The $y$-axis is in logarithmic scale. The black line represents the SRP and drag scenario, the gray line the drag-only scenario.

\section{Numerical simulation}

The orbit evolution was obtained by integrating the secular variation of Keplerian elements obtained through an averaging technique, given by Eqs. (16). In this subsection we verify this approximation, by using numerical propagation of the SRP and drag dynamics expressed in Cartesian coordinates. In this way we can assess the accuracy of the semi-analytical method used in Section II and verify the validity of some techniques and assumptions adopted:

- Use of an averaging technique for computing the secular change in orbital elements from Gauss' equations in true anomaly and eccentric anomaly form;

- Neglecting the coupling between SRP and drag;

- Use of Eqs. (14) to compute the change in orbital elements due to drag which are valid up to the fourth order of eccentricity.

American Institute of Aeronautics and Astronautics 
The dynamical equations in Cartesian coordinates consider the solar radiation pressure, with solar eclipses, atmospheric drag and the Earth's gravity field:

$$
\left\{\begin{array}{l}
\frac{d \mathbf{r}}{d t}=\mathbf{v} \\
\frac{d \mathbf{v}}{d t}=-\frac{\mu_{\text {Earth }}}{\|r\|^{3}} \mathbf{r}+\mathbf{a}_{\mathrm{SRP}}+\mathbf{a}_{\text {Drag }}
\end{array}\right.
$$

with

$$
\begin{aligned}
& \mathbf{a}_{\text {Drag }}=-\frac{1}{2} \frac{c_{D} A_{\text {drag }}}{m} \rho v_{\text {rel }}^{2} \hat{\mathbf{v}}_{\text {rel }} \\
& \mathbf{a}_{\text {SRP }}=a_{\text {SRP }} \hat{\mathbf{r}}_{\text {Sun-Earth }}
\end{aligned}
$$

where $\hat{\mathbf{r}}_{\text {Sun-Earth }}$ is the Sun-Earth direction, considering uniform motion of the Earth around the Sun on a circular orbit (i.e. $\left.\lambda_{\text {Sun }}(t)=\lambda_{\text {Sun }}(0)+n_{\text {Earth-Sun }} t\right)$.

For consistency, also the propagation of the dynamics in Cartesian coordinates is terminated once the orbit perigee reaches the lower boundary allowed (i.e., $h_{p}=250 \mathrm{~km}$ ). For the integration of Eqs. (22)-(23) an adaptive step-size Runge-Kutta-Fehlberg integration scheme integrator is used with a six stage pair of approximation of the fourth and fifth order [33], with absolute and relative tolerance of $2.5 \times 10^{-14}$.

Fig. 15 shows one orbit evolution scenario taken from Fig. 11, with initial conditions $e=0.12$, $\omega-\lambda_{\text {Sun }}=110.55 \mathrm{deg}, h_{p}=662.8 \mathrm{~km}$ and $a=8044.9 \mathrm{~km}$. The numerical propagation using the dynamical equations in Cartesian coordinates (black line) is superimposed on the propagation through the semi-analytical method (gray line). Fig. 15b shows the first phase of the mission; note that the Cartesian integration fully describes the dynamical evolution, capturing also the periodic variation of the orbital elements and the discontinuities due to the eclipse phase (i.e., discontinuities in the black line). The gray line instead, only describes the average behavior over one orbit revolution, giving the long-term evolution (see the two lines in Fig. 15a). The two lines differentiate much more close to the decay phase (see Fig. 15c), although giving the same estimation of the orbit lifetime. In fact the orbit lifetime $T_{L L}$ computed with the semi-analytical method is 47.759 days, compared to 47.773 days of the propagation in Cartesian coordinates.

Fig. 16a compares the numerical propagation through the semi-analytical formulae (gray line) with the integration in Cartesian coordinates (black line) for all the solutions represented in Fig. 11. For all the solution the averaged evolution gives a good estimate with that obtained by numerical integration of the equations of motion in Cartesian coordinates. The computational time required for the orbit propagation using the semi analytical method is two orders of magnitude lower than the computational time required for the integration in Cartesian coordinates. 


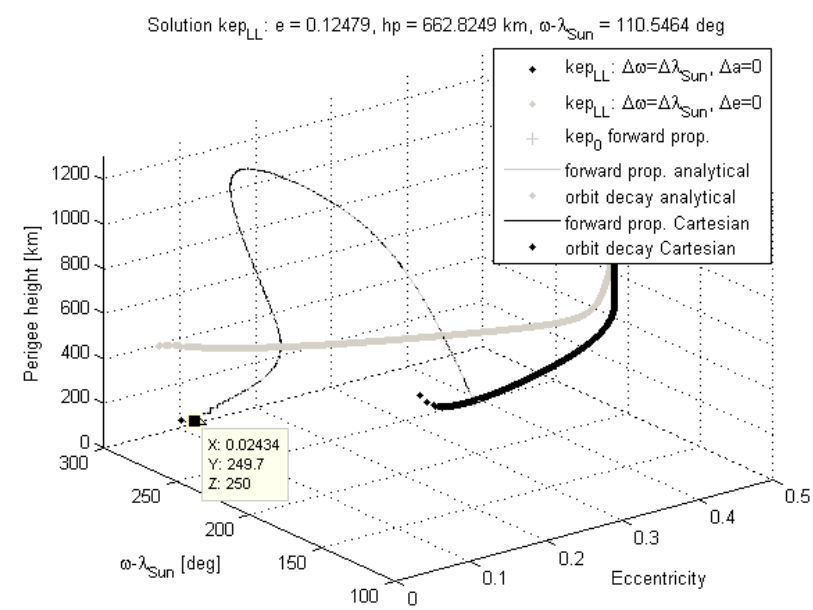

a)

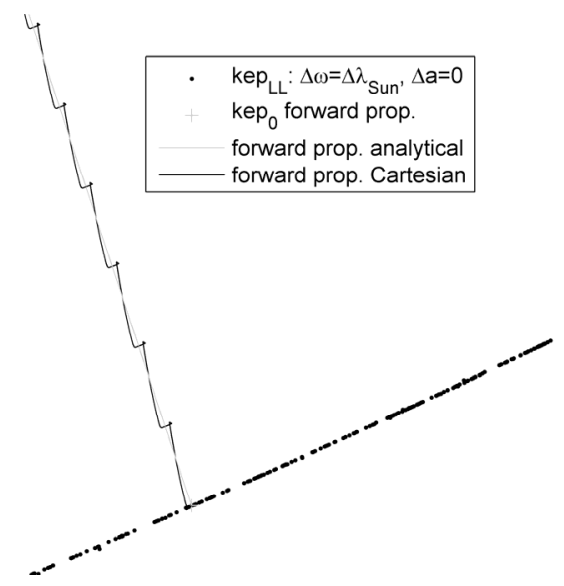

b)

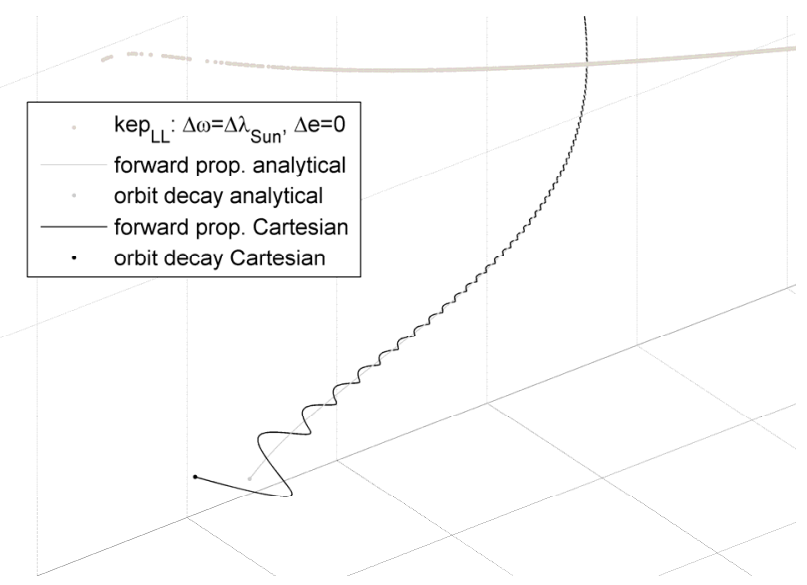

c)

Fig. 15 Numerical propagation of one solution for SpaceChip 3 in Cartesian coordinates from initial conditions with $\Delta a=0$ and $\Delta \omega=\Delta \lambda_{\text {sun. }}$. The orbital parameters of the initial orbit are $e=0.12, h_{p}=662.8 \mathrm{~km}, \omega$ $\lambda_{\text {Sun }}=110.55 \mathrm{deg}, a=8044.9 \mathrm{~km}$ and the orbit lifetime of the long-lived orbit is $T_{L L}=47$ days. The black line represents the Cartesian propagation, the gray line represents the semi-analytical propagation. a) Long-time orbit evolution, b) zoom on the initial phase, and c) zoom on the final decay phase. 


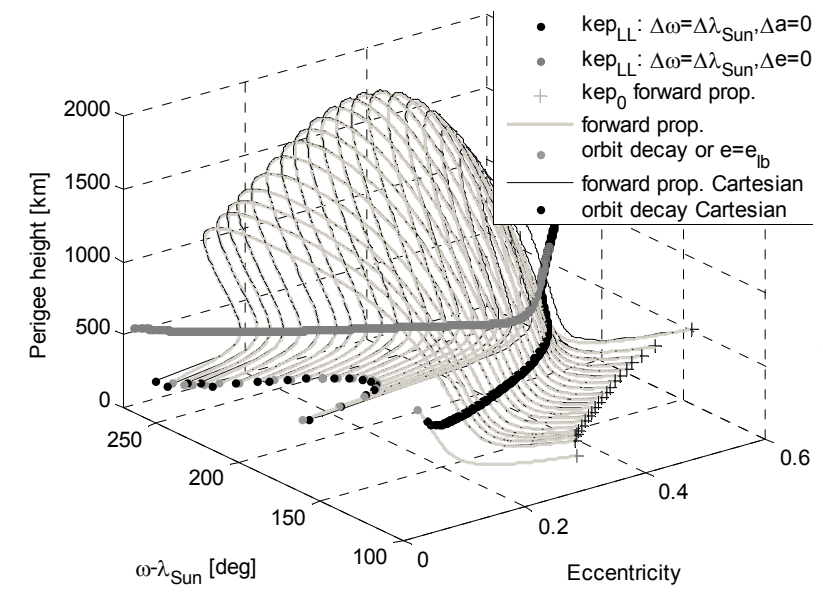

a)

Fig. 16 Numerical propagation of the solutions for SpaceChip 3 in Cartesian coordinates from initial conditions with $\Delta a=0$ and $\Delta \omega=\Delta \lambda_{\text {Sun. }}$. The black lines represent the Cartesian propagation, the gray lines represent the semi-analytical propagation. a) Propagation in the phase-space, and b) Orbit lifetime.

\section{B. Region $e \geq e^{*}$}

When we consider an initial condition vector which straddles the bifurcation region, the long-term evolution of the orbit presents an interesting behavior. As before, it is convenient to propagate the dynamics backwards in time, starting from a vector of initial conditions selected from the set of solutions of the system Eq. (17) or of the system Eq. (18) with a value of eccentricity close to the bifurcation region. The backward integration is continued until the stopping condition Eq. (21) is met. Fig. 17 shows an example of this orbit evolution behavior; the selected condition on the long-lived orbit graph is:

$$
k e p_{L L}: e=0.44085, h_{p}=799.0144 \mathrm{~km}, a=1.2835 .8 \mathrm{~km}, \omega-\lambda_{\text {Sun }}=1.78 .5229 \mathrm{deg}
$$

and $h_{p \text {,up }}$ was fixed at $3000 \mathrm{~km}$. Note that the choice of $h_{p \text {,up }}$ is arbitrary, as it is set only to fix a limit on the backward integration. The final state of the backward integration (black '+' symbol in Fig. 17) is used as the initial condition for the forward integration, until one of the conditions in Eq. (20) is met. As can be seen from Fig. 17a and the two projections in Fig. 17b and c, the long-term evolution in the phase-space diagram is characterized by a librational and progressively decaying motion around the equilibrium line which represents the solution of Eq. (17); i.e., the spacecraft performs quasi-closed loops in the eccentricity- $\left[\omega-\lambda_{\text {Sun }}\right]$ plane around the equilibrium point at which $\left\{\Delta a_{2 \pi}=0, \Delta e_{2 \pi}=0, \Delta \omega_{2 \pi}=\Delta \lambda_{\text {Sun, } 2 \pi}\right\}$. With respect to the SRP only case (see [19]) the loops do not close completely as they become smaller, while their centre point moves along the equilibrium line towards increasing values of eccentricity, i.e., the spacecraft describes a spiral in the phase-space. The orbit perigee oscillates around the Sun-line while the orbit stretches and contracts due to the oscillation both in eccentricity and semi-major axis. Fig. 18 depicts the evolution under SRP and drag in terms of orbit shape. Over one single loop in Fig. 17, the orbit librates as represented in Fig. 18a (orbit 1, orbit 2, orbit 3, orbit 4). Due to the effect of drag, a constant decaying motion is superimposed on the librational motion (see Fig. $18 \mathrm{~b}$ orbit 5 , orbit 6 , orbit 7 , orbit 8 ). This is clearly visible in Fig. 17b and Fig. 17d; the librational loops (due to the effects of SRP) become progressively smaller (i.e., the deviation from the centre value of eccentricity, $\omega-\lambda_{\text {sun }}$, and perigee height decreases) due to the effect of atmospheric drag. In particular from Fig. 17e, it is possible to infer that the effect of drag is almost negligible over the major part of the librational loop and becomes predominant in the arc of the loop when the perigee reaches its local minimum. In correspondence to the local minima of the perigee height, which oscillates due to SRP, the spacecraft experiences a rapid drop in orbit energy (see the step-fall in the semi-major axis in Fig. 17e), therefore the 
following librational loop will be centered on a point with a lower semi-major axis (i.e., lower value of the averaged orbit energy over the overall librational loop). In Fig. 17e the forward propagation due to SRP and drag (black line) is compared with the orbit evolution subjected to SRP only (bold black line). In the latter case the motion is exclusively librational (i.e., the spacecraft perpetually travels over the initial loop). Note that the inclination of the librational loop under SRP only, visible in the eccentricity - semi-major axis projection, is due to the asymmetry in geometry due to eclipses. In fact, in the presence of Earth's shadow the secular variation of semi-major axis is different than zero; therefore, the orbit energy is not conserved but the average energy, over one librational loop, is conserved.

The higher the equilibrium value of the semi-major axis (i.e., centre point of the librational loop), the lower is the excursion in semi-major axis over one loop. For a value of semi-major axis sufficiently high, the effect of eclipses can be neglected, and the SRP model (with eclipses) falls into the model used by Oyama et. al. (without eclipses) for which $\frac{d \bar{a}}{d t}$ SRP can be considered to be zero [19].

In Fig. 17 the orbit evolution under the effect of drag-only is shown (gray line). The initial condition for the forward propagation was set equal to the point of the forward propagation with SRP and drag (black line) where the first local minimum of perigee height is reached (gray ' + ' symbol).

When the effect of SRP cannot be exploited $\left(c_{R}=0\right)$, the orbit evolution follows a rotational motion where $\omega-\lambda_{\text {Sun }}$ continuously decreases (see Fig. 17a and Fig. 17c). The evolution of eccentricity and perigee height are also different; the orbit becomes increasingly circular while the perigee altitudes stays almost constant (see Fig. 17b). Once the orbit eccentricity becomes zero, the orbit radius starts to shrink quickly until the final decay in the lower atmosphere (gray ' $x$ ' symbol). In the SRP and drag scenario the final (and very fast) leg of the orbit decay lies within the $\omega-\lambda_{\text {Sun }}$ domain bounded by the two branches of the solutions of systems Eq. (18) and Eq. (19). Therefore, the bifurcation region can be seen as a sink for all the librational motion trajectories that design at higher values of semi-major axis.

The oscillation in perigee and apogee altitude through the orbit evolution is shown in Fig. 17f (black line) and is compared to the drag-only scenario (progressive constant decrease of the apogee, while the perigee height tends to remain constant), represented by the continuous gray line, and the SRP only scenario (the apogee and perigee continuously oscillate between their minimum and maximum value), represented by the bold black line.

Finally, Fig. 19 compares the time evolution of the Keplerian elements, under the effect of SRP and drag (black line), drag only (gray line), and SRP only (light gray line). The perigee oscillation due to SRP is damped by the presence of drag. The atmospheric drag, mainly experienced in the region around $700 \mathrm{~km}$ (for SpaceChip 3), causes the decrease of the maximum value of the perigee that is reached over each librational loop. The minimum perigee (in analogy with the orbit perigee of the drag only case), instead, tends to remain constant until the last part of the decay phase (see Fig. 19a). The semi-major axis behavior in time is characterized by a periodic motion (with the period of one librational loop that is different from the period of one single orbit) due to SRP, plus a quasi-constant energy decrease due to drag (see Fig. 19b). Note that the orbit life time with SRP and drag is around 28 years, compared to the 11.3 years of the drag-only scenario. Therefore, long-lived orbits can be designed where asymmetric solar radiation pressure can balance energy dissipation due to air drag. In addition, the effect of atmospheric drag can be exploited to ensure the end-of life decay of SpaceChips, thus preventing long-lived orbit debris. As already pointed out for Fig. 17d, the orbit perigee oscillates around the Sun direction $\left(\omega-\lambda_{\text {Sun }}=\pi\right)$ and the oscillation are damped by atmospheric drag (see Fig. 19c). In the drag-only scenario, $\omega-\lambda_{\text {Sun }}$ is continuously decreasing as shown in Fig. 17a and Fig. 17c. As can be seen in Fig. 19d and Fig. 17b, the eccentricity oscillates around the value that satisfies system Eq. (17) for a given semi-major axis and $\omega-\lambda_{\text {Sun }}=\pi$. 

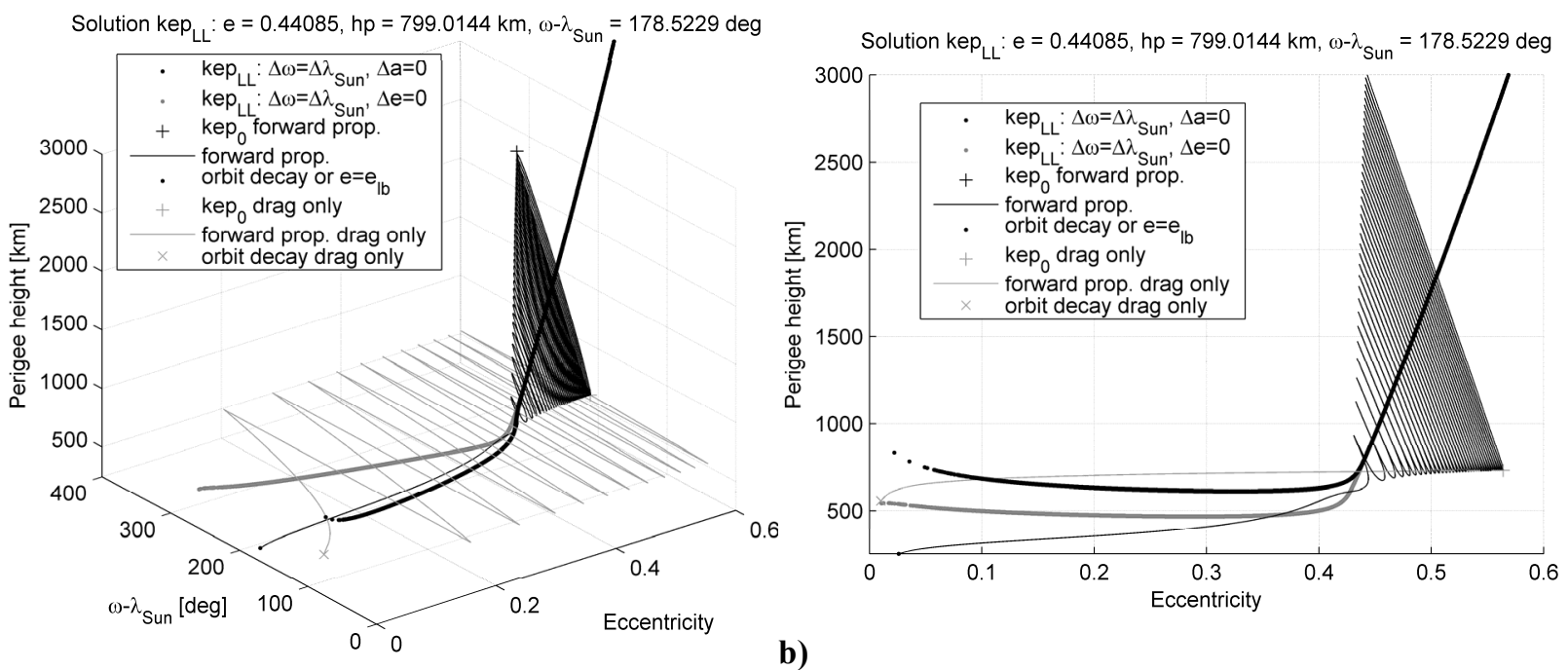

b)

a)
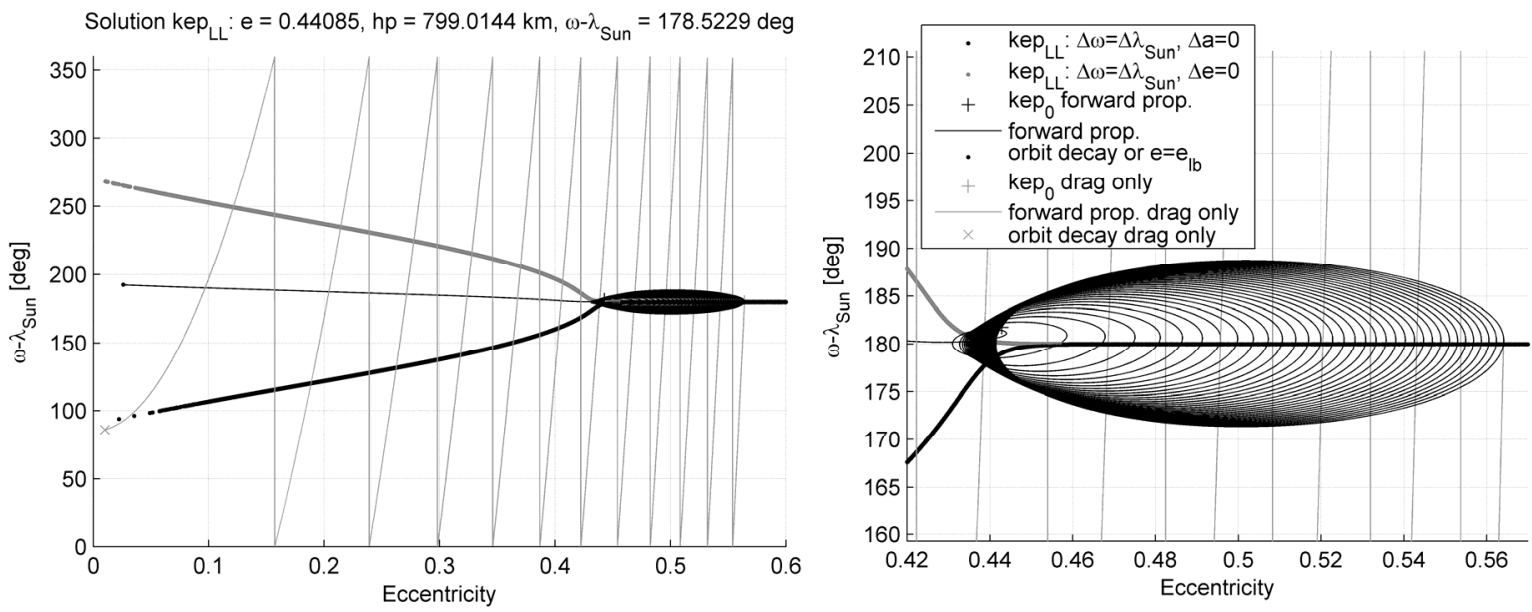

c)

d)
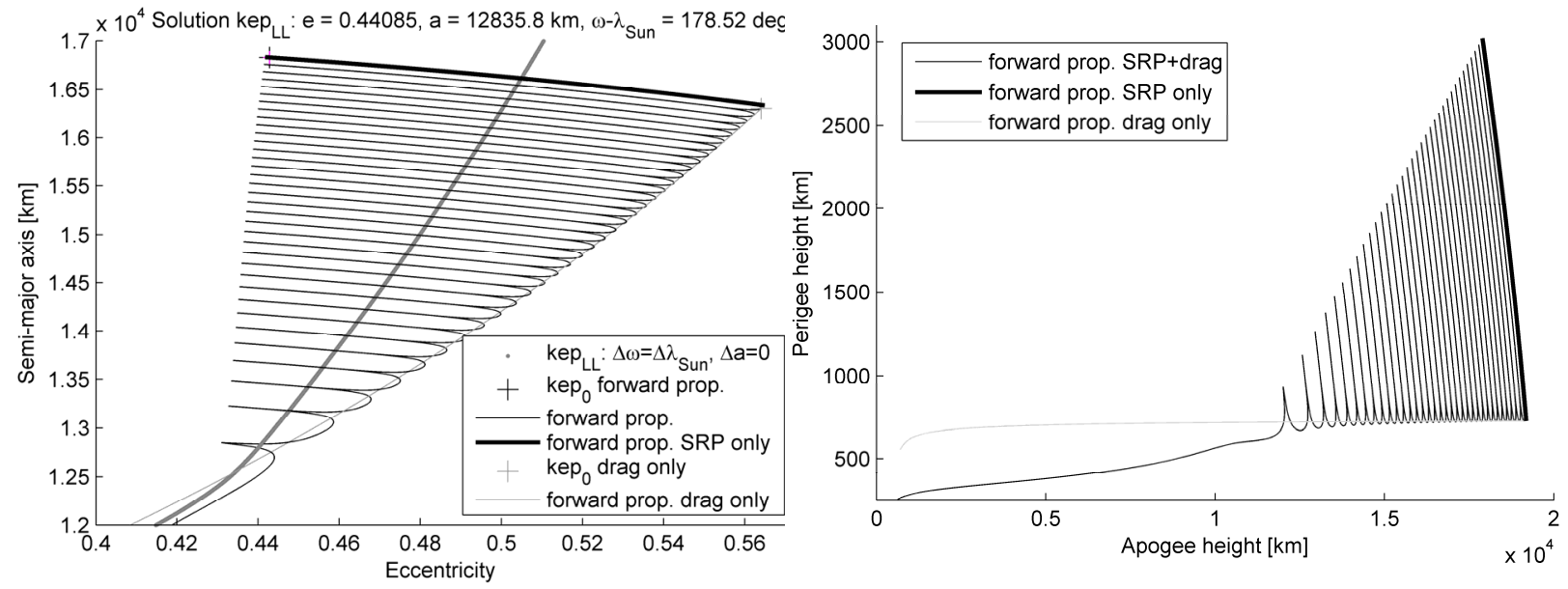

e)

f)

Fig. 17 Long-term orbit evolution for SpaceChip 3 from a vector of initial conditions for librational 
motion. The black lines represent the SRP and drag scenario, the gray lines the drag-only scenario, the bold black lines the SRP-only scenario. The '+ symbol' represents the initial condition for the forward propagation, the ' ' symbol represents the end-condition for the SRP and drag case, the ' $x$ symbol' represents the end-condition for the drag-only case. a) 3D view in the phase-space with perigee height on the z-axis, b) $2 \mathrm{D}$ view in eccentricity - perigee height, c) $2 \mathrm{D}$ view in eccentricity $-\omega$ - $\lambda_{\text {Sun }}$ d) $\mathrm{zoom}$ of $2 \mathrm{D}$ the view in eccentricity $-\omega-\lambda_{\text {Sun }}$ to appreciate the librational motion, d) $2 D$ view in eccentricity - semimajor axis, and e) oscillation of the apogee and the perigee height.

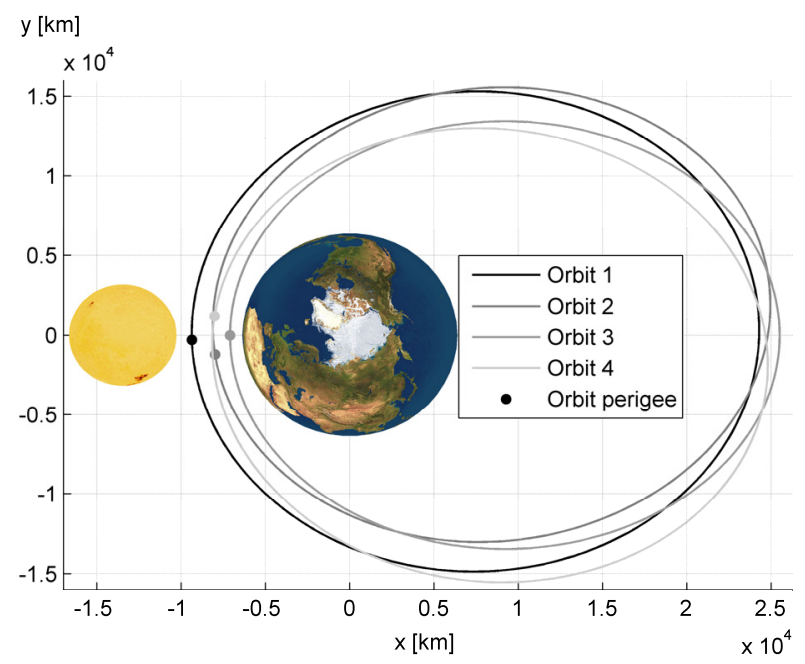

a)

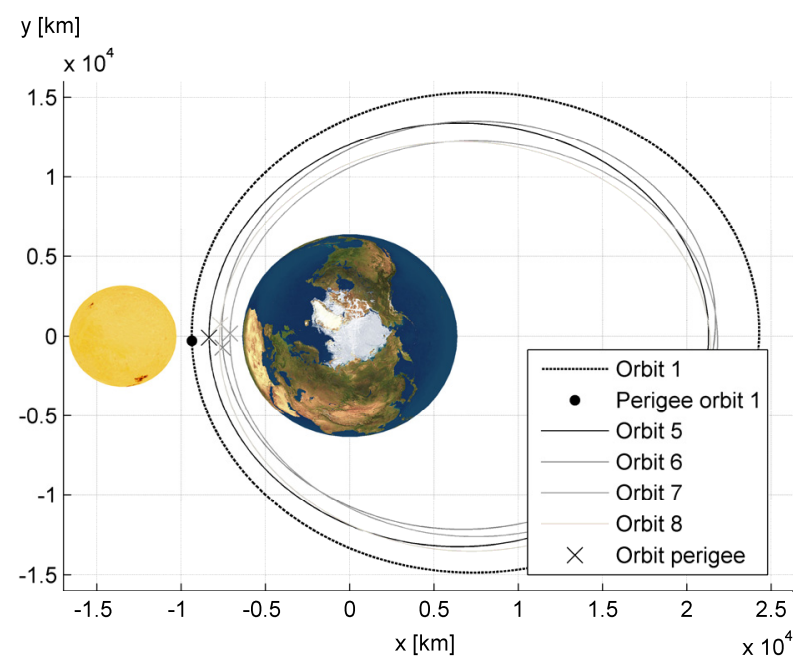

b)

Fig. 18 Librational and progressively decaying motion due to SRP and drag. a) The orbit perigee oscillates around the Sun-line and the orbit shape changes due to the oscillation in eccentricity and semimajor axis. b) Due to the effect of drag the orbit perigee is subject to a secular decrease.

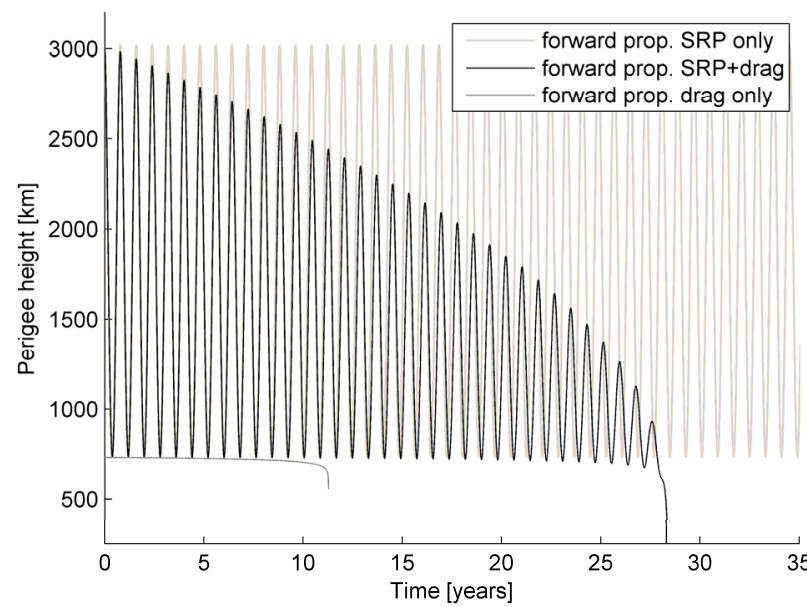

a)

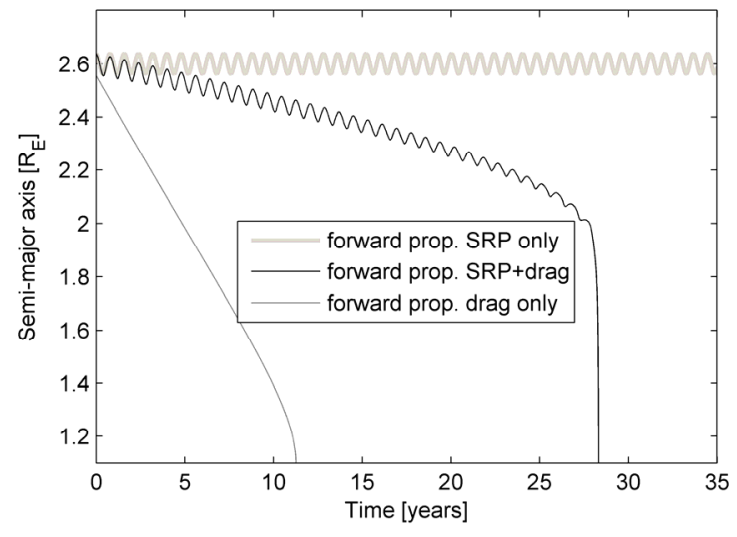

b) 


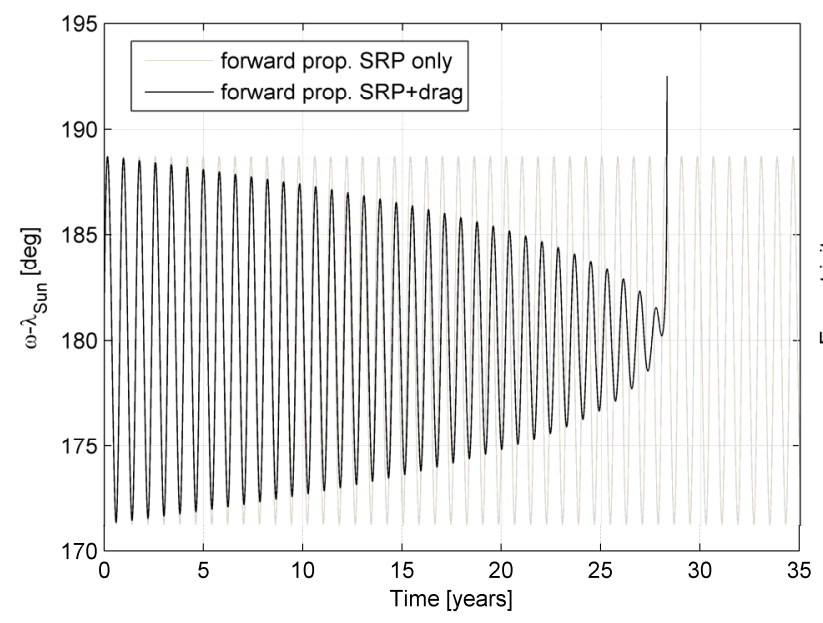

c)

Fig. 19 Evolution of the Keplerian elements for SpaceChip 3, under the effect of SRP and drag (black line), drag-only (gray line), and SRP-only (light gray line). a) Perigee height, b) semi-major axis in Earth radii, c) angular displacement with respect to the Sun direction, $\omega-\lambda_{\text {Sun }}$, and d) eccentricity.

The orbit evolution analyzed for the selected solution in Fig. 17-Fig. 19 can be reproduced selecting any point straddling the bifurcation region of the long-lived orbits graph (see Fig. 10). This procedure is shown in Fig. 20; different vectors of initial conditions $k e p_{L L}$ belonging to the branches Eq. (18) and Eq. (17) of the long-lived orbits graph are selected, and ordered in increasing eccentricity. These sets of orbital elements are indicated in Fig. 20a with a 'o' symbol and gray scale. Starting from those points, the forward and backward propagation of the SpaceChip orbit, under the effect of SRP and drag can be seen. The stopping criterion for the forward integration is defined by Eq. (20), whereas the stopping criterion for the backward integration is defined by Eq. (21), with $h_{p \text {, up }}=8000 \mathrm{~km}$ for this simulation. The first two solutions represented in Fig. 20 (darker gray lines) show the same behavior of the solutions in Fig. 11: the backward propagation from the initial condition vectors $k p_{L L}$ terminates without performing any librational loop, because the stopping criterion $e \geq 0.8$ or $h_{p} \leq 250 \mathrm{~km}$ is encountered (see the ' + ' symbol in Fig. 20d). The forward propagation shows an increase of the perigee altitude, because of SRP, while $\omega-\lambda_{\text {Sun }}$ is less than $\pi$; afterwards the perigee altitude starts decreasing, until encountering the surface $\Delta e_{2 \pi}=0$ (visible in Fig. 9 for SpaceChip 1). At that point the trajectory in the phase-space presents an elbow which indicates that the final fast decay phase has started, due to the predominant effect of atmospheric drag.

The third solution portrayed in Fig. 20, instead, performs, in the backward evolution, a librational loop around the equilibrium line Eq. (17), until meeting the stopping condition $h_{p} \geq h_{p \text {, up }}$ (see the '+' symbol in Fig. 20d). Fig. 20d highlights that the orbit evolution analyzed in Section V.A (see Fig. 11) and Section V.B (see Fig. 17) do not represent a different behavior; in fact, they can be seen as a continuation with increasing values of the eccentricity of the initial condition vector $k_{e p}$ selected on the long-lived orbits graph. The higher the value of eccentricity of the vector $k e p_{L L}$ (we are selecting as initial condition vectors points belonging to the branch Eq. (18) and Eq. (17), sorted in ascending order based on the value of the eccentricity), the higher number of librational loops appears in the backward propagation, and the smaller the radius of the cone that the trajectory will perform in the phase-space around the solution set of stable equilibrium Eq. (17), as can be seen in Fig. 20a. In other terms, the orbit evolution identified by vectors kep $_{L L}$ with higher values of eccentricity will be bounded within the cone described by the trajectory evolution identified by the vector of initial conditions $k e p_{L L}$ at the edges of the bifurcation region. This means, furthermore, that if we select any vector of initial conditions inside this bifurcation cone (which represent the boundary), the long-term evolution will be enclosed in the cone itself, and the motion will be librational and progressively decaying. This is highlighted in Fig. 20b, where an initial condition vector is chosen inside the cone, and the trajectory is propagated forward in time (black line). Moreover, it is interesting to note that all these solutions evolve towards their end-of-life through the bifurcation region, which can be seen (as already pointed out 
for Fig. 17) as a sink for all librational-decaying trajectories which generate at higher values of the semi-major axis and eccentricities inside the bifurcation cone. Analogously, as shown in Section V.A (see Fig. 11), all the trajectories generated on the branch Eq. (18) $\left(\Delta a_{2 \pi}=0\right.$ and $\left.\Delta \omega_{2 \pi}=\Delta \lambda_{\text {Sun, } 2 \pi}\right)$, or the branch Eq. (19) ( $\Delta e_{2 \pi}=0$ and $\left.\Delta \omega_{2 \pi}=\Delta \lambda_{\text {Sun, } 2 \pi}\right)$ will evolve towards their end-of-life within the region of $\omega-\lambda_{\text {Sun }}$ bounded by the two branches.

Going back to the spiral trajectories shown in Fig. 20a, the higher the value of the eccentricity in the vector $k e p_{L L}$, the higher number of librational loops the spacecraft will perform from an upper value of the perigee altitude, until the final decay. As a consequence, the orbit lifetime from a fixed altitude to the decay is higher, as shown in Fig. 21. Fig. 21 shows the lifetime of the orbit, in logarithmic scale, with the eccentricity of the initial condition vector $k e p_{L L}$ identifying each trajectory. By comparing different spiral trajectories in Fig. 20a, it can be seen that when the trajectory arc in the phase-space between two consecutive librational loops (due to the effect of SRP) is more extended, the influence of the drag is higher on that arc, thus determining the satellite's lifetime.

For the same reason we can infer that if we select different initial conditions at a certain fixed semi-major axis, with different values of eccentricity, the orbit lifetime will be higher as the initial eccentricity will be chosen close to the equilibrium eccentricity for that value of semi-major axis and it will decrease going in a radial direction from the equilibrium value of the eccentricity). In fact, at fixed semi-major axis, the smaller the radius of the librational loop, the lower is the effect of drag and limited to a smaller part of the librational loop. To the limit, when a vector kep $_{L L}$ is chosen along the solution set Eq. (17) (i.e., the radius of the librational loop is zero) with a sufficiently high value of semi-major axis, the orbit lifetime will be infinite, as the spacecraft is stable in that position, under the influence of SRP and the non-conservative effect of drag can be neglected. These solutions (without considering Earth's shadow) were found by McInnes et al. for a solar sail mission application [18].

The decrease of the orbit lifetime is clearly a function of the increasing influence of atmospheric drag as can be seen in Fig. 24. For this test, different initial conditions have been selected with the same eccentricity, $\omega-\lambda_{\text {Sun }}$ and decreasing values of perigee height; those initial condition are indicated in Fig. 22 with a ' + ' symbol and gray color scale. The trajectory has been propagated forward in time, under the influence of SRP and drag (continuous line) and drag-only (dashed line) and is represented in Fig. 22. Fig. 23a and Fig. 23b show the time evolution of the perigee altitude and the semi-major axis for the different trajectories, with SRP and drag and drag-only, while Fig. 24 shows the orbit lifetime for the two scenarios, as a function of the initial perigee height of the orbit propagation.
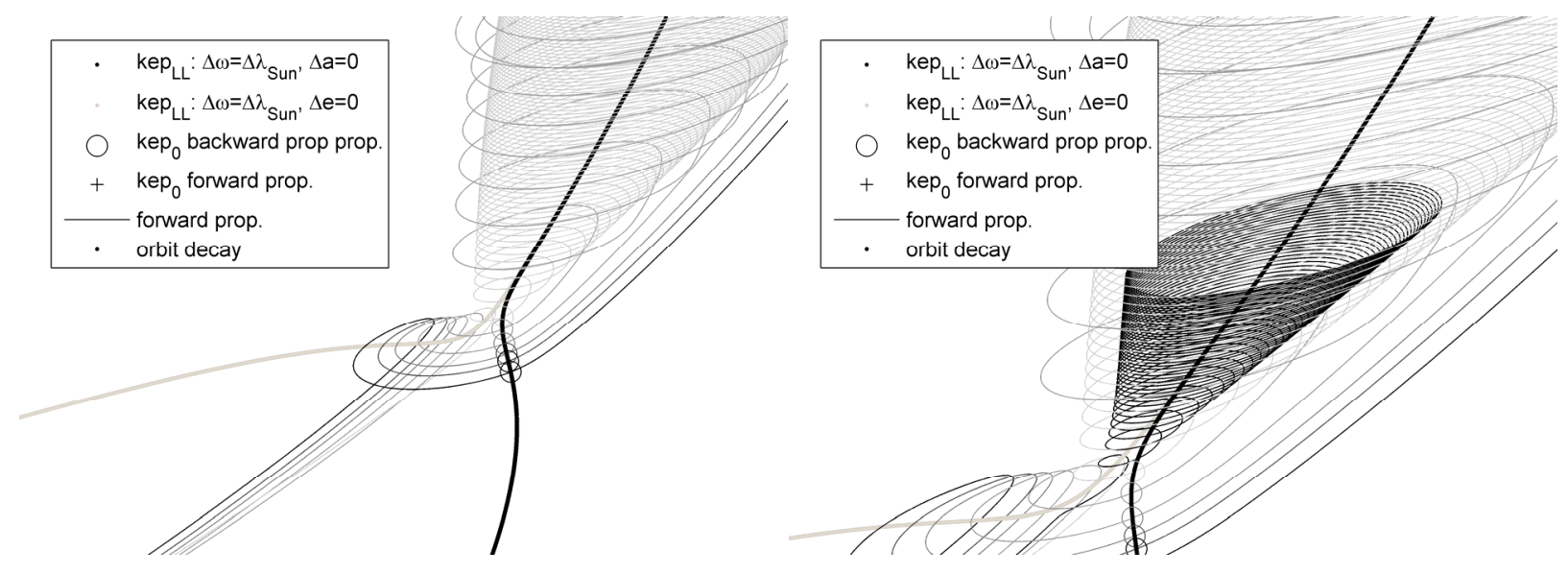

a)

b) 

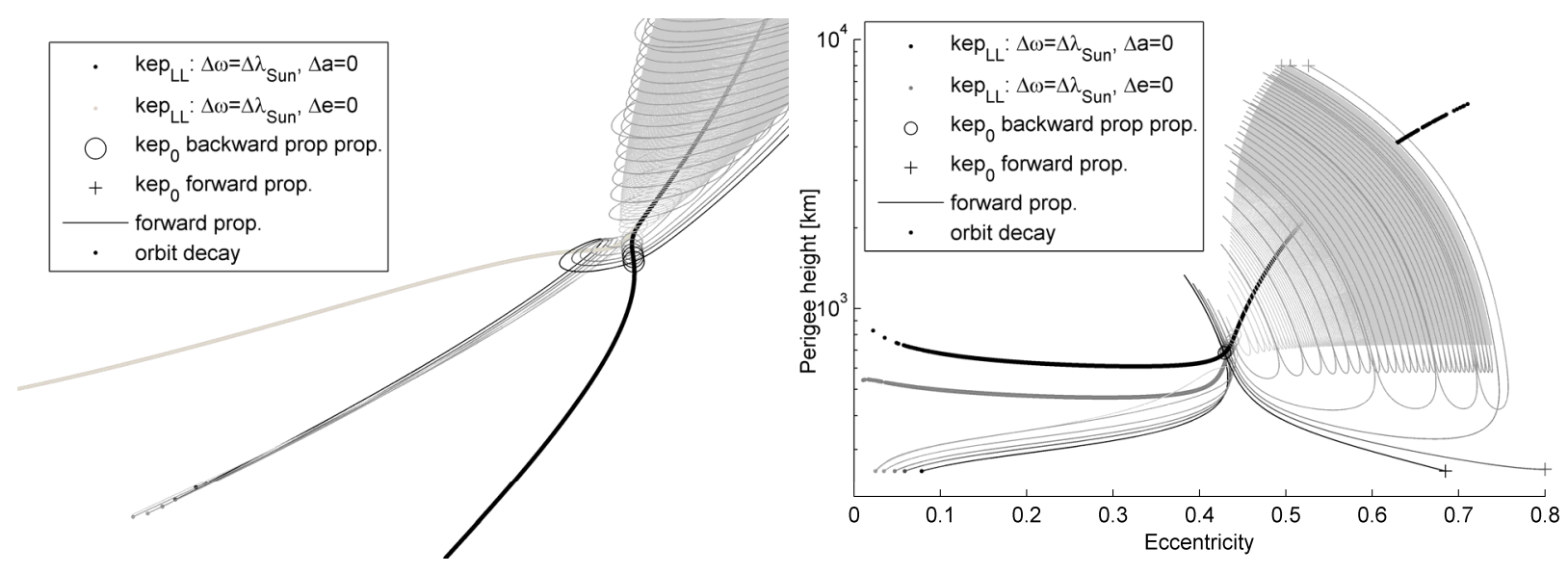

c)

d)

Fig. 20 Long-term orbit evolution for SpaceChip 3 from set of vectors of initial conditions on the bifurcation region under the effect of SRP and drag. A gray color scale is used to differentiate the different initial conditions of the long-lived orbits graph. The 'o' symbol represents the initial condition on the long-lived orbits graph for the backward propagation, the '+' symbol represents the stopping point of the backward propagation and the initial condition for the forward propagation, the '.' symbol represents the end-condition for the forward propagation. a) Zoom on the points selected on the long-lived orbits graph. The axes of the graph are eccentricity, $\omega-\lambda_{\text {Sun }}$, and semi-major axis. b) Trajectory enclosed in the bifurcation cone. The axes of the graph are eccentricity, $\omega-\lambda_{\text {Sun }}$, and semi-major axis. c) Zoom on the decay phase. The axes of the graph are eccentricity, $\omega-\lambda_{\text {sun }}$, and semi-major axis. d) $2 \mathrm{D}$ view of the solutions in eccentricity - perigee height in logarithmic scale.

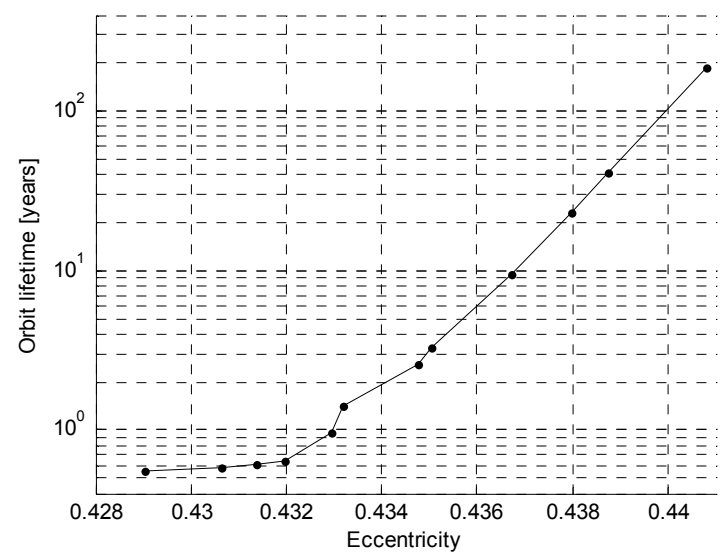

Fig. 21 Orbit lifetime of the solutions for SpaceChip 3 shown in Fig. 20. 

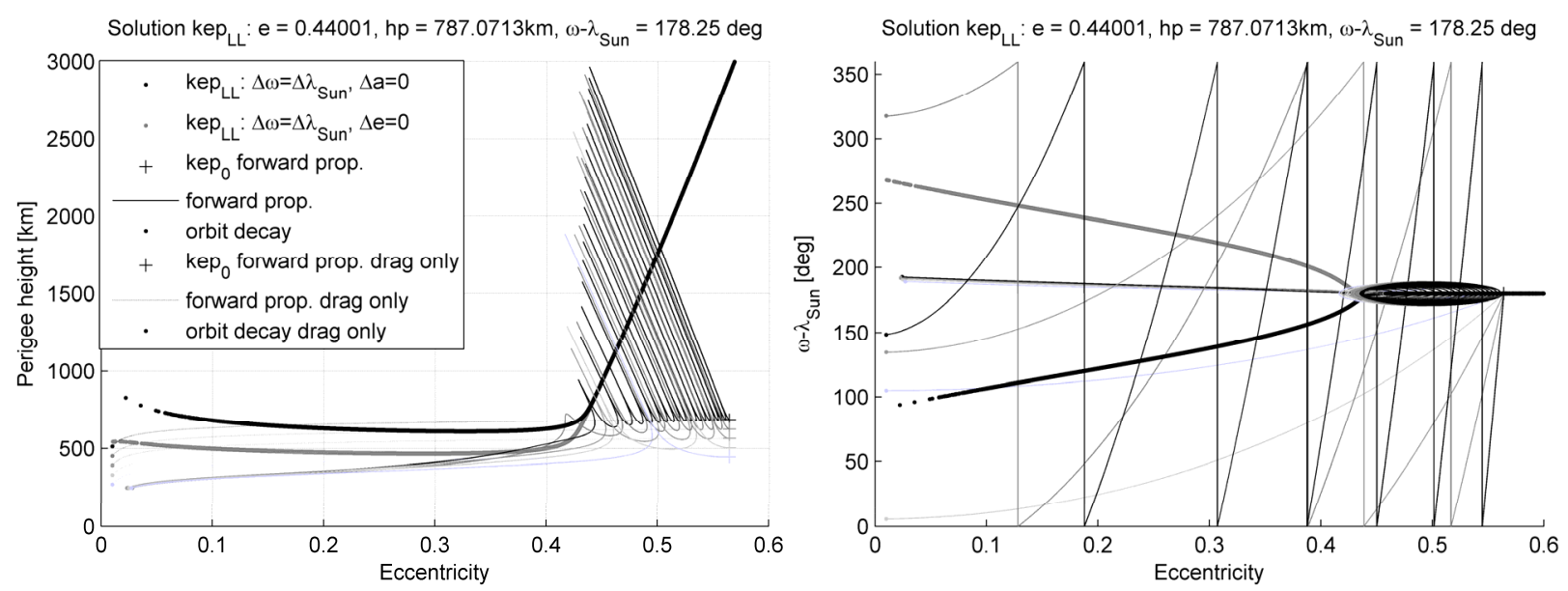

a)

b)

Fig. 22 Long-term orbit evolution for SpaceChip 3 from set of vectors of initial conditions with the same eccentricity and $\omega-\lambda_{\text {Sun }}$, and different perigee height (gray color scale), under the influence of SRP and drag (continuous line) and drag-only (dashed line). The '+' symbol represents the initial condition for the forward propagation, the '.' symbol represents the end-condition for the forward propagation (i.e., orbit decay). a) 2D view of the solutions in eccentricity - perigee height, and b) 2D view of the solutions in eccentricity $-\omega-\lambda_{\text {Sun }}$.
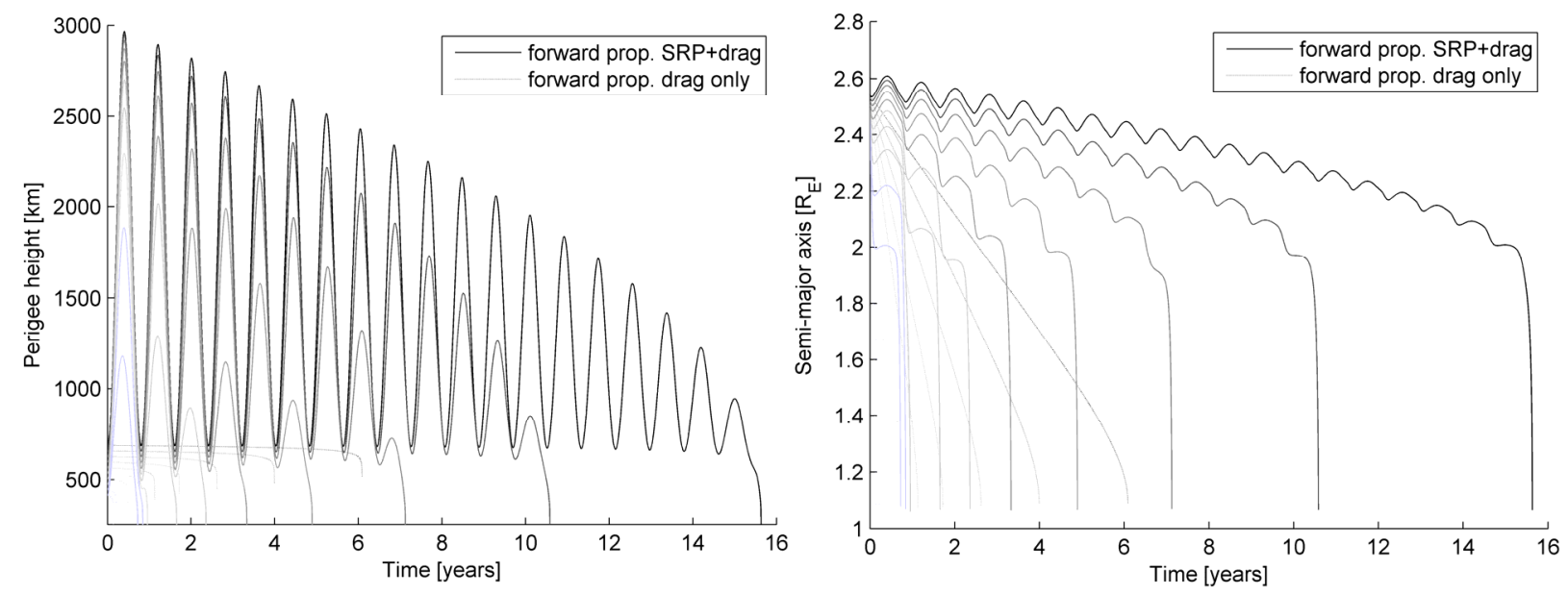

a)

b)

Fig. 23 Evolution of the Keplerian elements for the solutions in Fig. 22. The continuous line represents the SRP and drag scenario, the dashed line represents drag-only scenario. a) Perigee height, b) semimajor axis in Earth radii. 


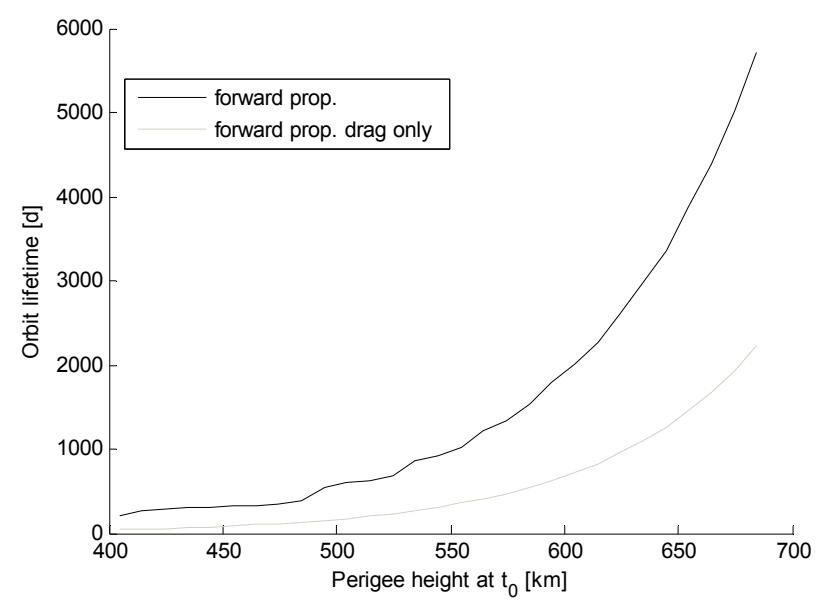

Fig. 24 Orbit lifetime of the solutions for SpaceChip 3 shown in Fig. 22. The black line is the SRP and drag case, the gray line is the drag-only case.

Finally, we point out that there exists a wider set of initial conditions for which the long-term evolution is characterized by a librational and progressively decaying motion. Those orbits can be identified as those which intersect the surface $\Delta \omega_{2 \pi}=\Delta \lambda_{\text {Sun, } 2 \pi}$, as shown in Fig. 25 and Fig. 26 (in correspondence to the intersection with the surface $\Delta \omega_{2 \pi}=\Delta \lambda_{\text {Sun, } 2 \pi}$ the partial derivative with respect to $\omega-\lambda_{\text {Sun }}$ is zero as can be seen in Fig. 26b). In order to identify those orbits, the corresponding initial condition was chosen with $\omega-\lambda_{\text {Sun }}=\pi$ and, fixing a value of semimajor axis, the eccentricity must be lower that the eccentricity for which Eq. (17) is satisfied. These points are represented with a '+' symbol in Fig. 25 and Fig. 26. Starting from these condition the forward and backward propagation were performed, to show the long-term behavior in the phase-space. As noted, these orbits are characterized by a librational and progressively decaying motion, however the decay phase will not be enclosed in the bifurcation cone shown in Fig. 20b. All these trajectories, similarly to the trajectories presented in Fig. 11, present an elbow in the phase-space in correspondence of meeting the surface $\Delta a_{2 \pi}=0$ or $\Delta e_{2 \pi}=0$. 


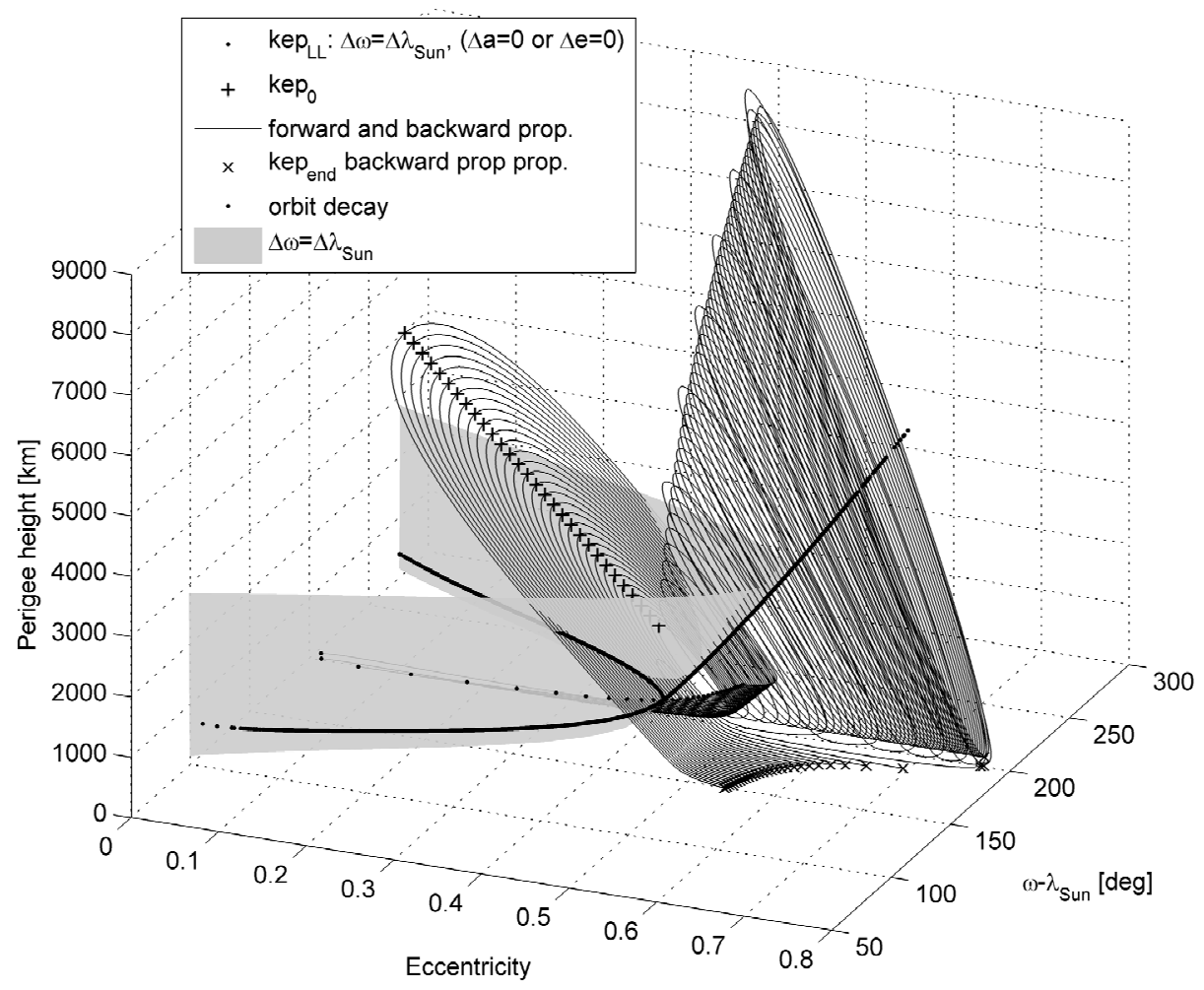

Fig. 25 Long-term orbit evolution for SpaceChip 3 for condition with librational and progressively decaying motion.
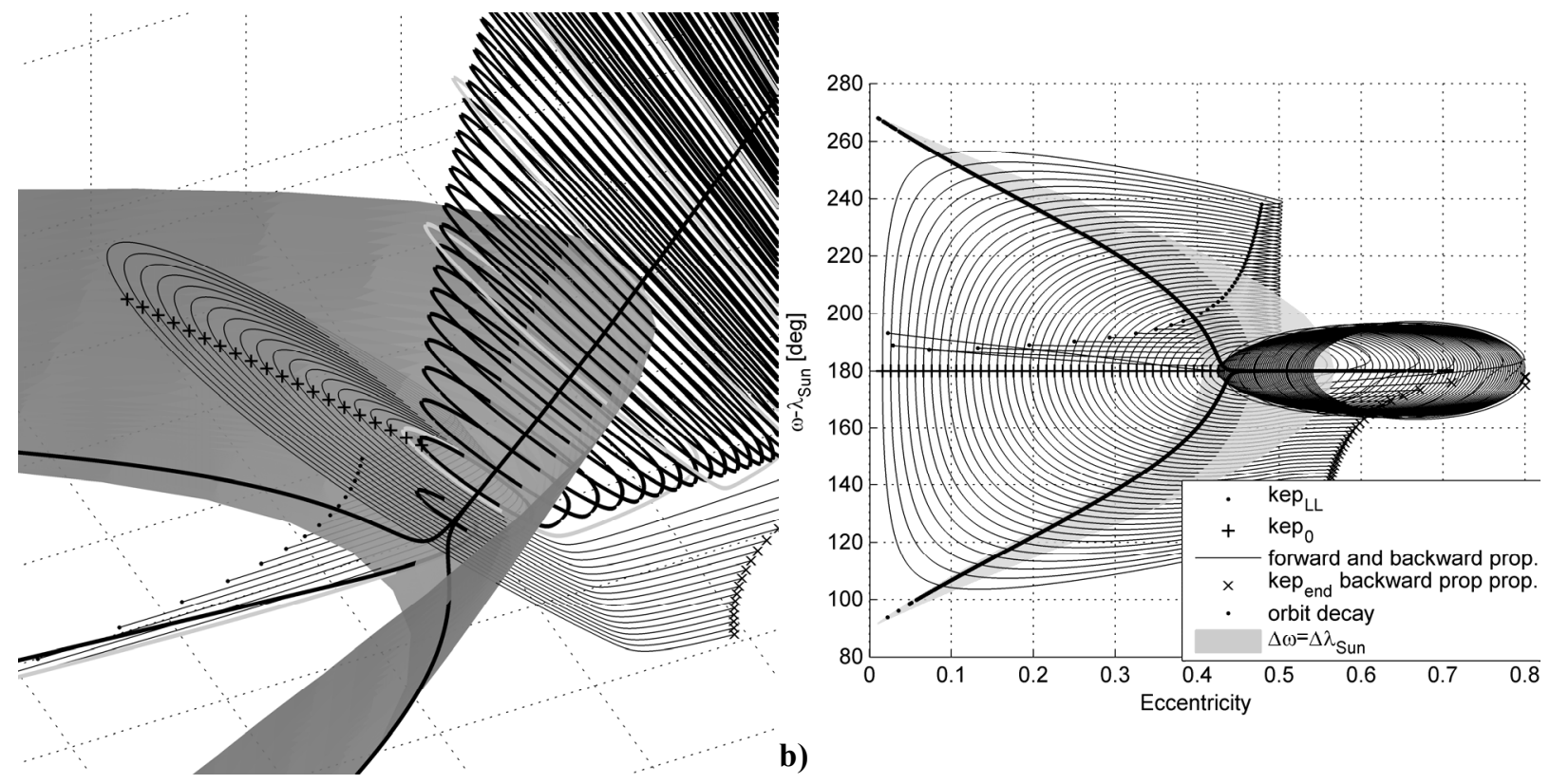

b)

a)

Fig. 26 Long-term orbit evolution for SpaceChip 3 for condition with librational and progressively decaying motion. a) Zoom on the bifurcation region, and b) $2 \mathrm{D}$ view in eccentricity $-\omega-\lambda_{\text {Sun }}$. 
The higher the area-to-mass ratio, the more extended is the region of the phase-space domain where the motion under SPR and drag is librational, thus enlarging the sun-synchronous mission possibilities. Outside this region, instead, the motion is rotational (where $\omega-\lambda_{\text {Sun }}$ continues to decrease). For a more extensive definition of rotational motion, see Ref. [19].

\section{Mission applications}

McInnes at al. [18] showed that a solar sail with a characteristic acceleration of $0.138 \mathrm{~mm} / \mathrm{s}^{2}$ can be used for a geomagnetic tail mission on a $10 \times 30$ Earth radii, to artificially precess the apse line in Sun-synchronous manner. Oyama et al. [19] extended the analysis in the phase-space to study the global behavior of the solar sail orbits around the Earth; with respect to McInnes at al., a more extended useful region of the space-domain was identified for increasing the scientific return of the mission. In the present paper it was shown that the Sun-synchronous precession of the apse line can be artificially obtained also with a SpaceChip of $1 \mathrm{~cm}^{2}$ area and different thickness values, at different technology readiness levels of current nano-fabrication technologies. This concept can be adapted to enhance the return of a GEOSAIL mission. A swarm of SpaceChips can be used as distributed nodes of a network in the useful region of the phase-space, to obtain a spatial and temporal map of the geomagnetic tail, as the concept of Kilo-Satellite constellation concept, proposed by Petschek et al. [34]. The coupled effect of atmospheric drag and solar radiation, with asymmetry due to eclipses can be exploited to define the orbit lifetime and to assess and design the disposal of the SpaceChips at the end of mission. Importantly, the short life-time of high area-to-mass spacecraft can be greatly extended (and indeed selected) through the interaction of energy gain from asymmetric solar radiation pressure and energy dissipation due to drag.

\section{Conclusions}

This paper analyzes the orbital dynamics of SpaceChips under the influence of solar radiation pressure and atmospheric drag. The high area-to-mass ratio, with respect to conventional spacecraft, allows the exploitation of the disturbances on the Keplerian motion as passive method to control orbit evolution without the use of active orbit control. The secular and long-period changes of Keplerian elements are computed through semi-analytic formulae. The initial conditions for long-lived orbits for SpaceChips are determined, by exploiting asymmetric solar radiation pressure to offset the effect of atmospheric drag. Through the long-term propagation of the orbit evolution we identified regions of the phase-space in which the motion of the spacecraft is librational and progressively decaying due to non-conservative energy losses due to drag. The asymmetric effect of solar radiation pressure allows a modulation of the orbit energy and angular momentum throughout a full librational loop and the sun-synchronous precession of the apse line. As a consequence, the orbit perigee oscillates between a minimum and a maximum value. The comparison with the drag-only and SRP-only scenario demonstrates that atmospheric drag acts as a damping on the oscillation due to SRP. When the orbit perigee passes through its minimum, the effect of drag becomes dominant. Subsequent librational loops evolve with smaller radius and librating around a center that moves towards lower values of semi-major axis and eccentricity. The bifurcation region near which the equilibrium solution under SRP and drag disappears can be seen as a sink for the librational motion trajectories generated at higher values of semi-major axis and eccentricities. Thus, the life-time of high area-to-mass spacecraft can be greatly extended (and indeed selected) through the exploitation of asymmetric solar radiation pressure, and the effect of drag can be exploited to obtain fast decay of these 'smart dust' devices in the terminal phase of the mission, dealing with issues related to end-of-life disposal and the creation of long-lived space debris from swarm of devices. For higher values of perigee height, a set of solutions is identified for which the orbit is in equilibrium under the effect of SRP only, and the influence of drag is negligible. These families of equilibrium and librational orbits are proposed as baseline for future SpaceChip swarm missions for geomagnetic tail observation; the high area-to-mass ratio allows an enlargement of the useful region of the domain for increasing the scientific return, and the low fabrication cost of these devices envisage their use for spatial and temporal distribution of the possible measurements.

\section{Acknowledgments}

This work was funded by the European Research Council, as part of project VISIONSPACE (227571). 


\section{References}

[1] Vladimirova, T., Xiaofeng, W. and Bridges, C. P., "Development of a Satellite Sensor Network for Future Space Missions," Proceedings of the Aerospace Conference, 2008 IEEE, 2008, pp. 1-10.

[2] Barnhart, D. J., Vladimirova, T. and Sweeting, M. N., "Very-Small-Satellite Design for Distributed Space Missions," Journal of Spacecraft and Rockets, Vol. 44, No. 6, 2007, pp. 1294-1306. doi: 10.2514/1.28678

[3] Warneke, B. A. and Pister, K. S. J., "Mems for Distributed Wireless Sensor Networks," Proceedings of the Electronics, Circuits and Systems, 2002. 9th International Conference on, Vol. 1, 2002, pp. 291-294.

[4] Atchison, J. A. and Peck, M. A., "A Passive, Sun-Pointing, Millimeter-Scale Solar Sail," Acta Astronautica, Vol. 67, No. 1-2, 2009, pp. 108-121. doi: 10.1016/j.actaastro.2009.12.008

[5] Shapiro, I. I., Jones, H. M. and Perkins, C. W., "Orbital Properties of the West Ford Dipole Belt," Proceedings of the IEEE, Vol. 52, No. 5, 1964, pp. 469-518.

[6] Hamilton, D. P. and Krivov, A. V., "Circumplanetary Dust Dynamics: Effects of Solar Gravity, Radiation Pressure, Planetary Oblateness, and Electromagnetism," Icarus, Vol. 123, No. 2, 1996, pp. 503-523. doi: 10.1006/icar.1996.0175

[7] Greenberg, R. and Brahic, A. (eds.), Planetary Rings, Space Science Series. University of Arizona Press, Tucson, 1984.

[8] Grotta-Ragazzo, C., Kulesza, M. and Salomao, P. A. S., "Equatorial Dynamics of Charged Particles in Planetary Magnetospheres,” Physica D: Nonlinear Phenomena, Vol. 225, No. 2, 2007, pp. 169-183. doi: 10.1016/j.physd.2006.10.009

[9] Gor'kavyi, N. N., Ozernoy, L. M. and Mather, J. C., "A New Approach to Dynamical Evolution of Interplanetary Dust,” Astrophysical Journal, Vol. 474, No. 1, 1997, pp. 496-496. doi: 10.1086/303440

[10] Howard, J. E., Horanyi, M. and Stewart, G. R., "Global Dynamics of Charged Dust Particles in Planetary Magnetospheres," Physical Review Letters, Vol. 83, No. 20, 1999, pp. 3993-3996. doi: 10.1103/PhysRevLett.83.3993

[11] Schildknecht, T., Musci, R. and Flohrer, T., "Properties of the High Area-to-Mass Ratio Space Debris Population at High Altitudes," Advances in Space Research, Vol. 41, No. 7, 2008, pp. 1039-1045. doi: 10.1016/j.asr.2007.01.045

[12] Anselmo, L. and Pardini, C., "Long-Term Dynamical Evolution of High Area-to-Mass Ratio Debris Released into High Earth Orbits," Acta Astronautica, Vol. 67, No. 1-2, 2010, pp. 204-216. doi: 10.1016/j.actaastro.2009.10.017

[13] Shapiro, I. I. and Jones, H. M., "Perturbations of the Orbit of the Echo Balloon," Science, Vol. 132, No. 3438, 1960, pp. 1484-1486. doi: 10.1126/science.132.3438.1484

[14] Musen, P., Bryant, R. and Bailie, A., "Perturbations in Perigee Height of Vanguard I," Science, Vol. 131, No. 3404, 1960, pp. 935-936. doi: 10.1126/science.131.3404.935

[15] Harwood, N. M. and Swinerd, G. G., "Long-Periodic and Secular Perturbations to the Orbits of Explorer 19 and Lageos Due to Direct Solar Radiation Pressure," Celestial Mechanics and Dynamical Astronomy, Vol. 62, No. 1, 1995, pp. 81-92. doi: 10.1007/BF00692070

[16] McInnes, C. R., Solar Sailing: Technology, Dynamics and Mission Applications, Springer-Praxis Books in Astronautical Engineering, Springer-Verlag, Berlin, 1999.

[17] Kawaguchi, J. i., Mimasu, Y., Mori, O., Funase, R., Yamamoto, T. and Tsuda, Y., "Ikaros - Ready for LiftOff as the World's First Solar Sail Demonstration in Interplanetary Space," Proceedings of the 60th International Astronautical Congress, IAC 2009, Daejeon, Korea, 2009.

[18] McInnes, C. R., Macdonald, M., Angelopolous, V. and Alexander, D., "Geosail: Exploring the Geomagnetic Tail Using a Small Solar Sail," Journal of Spacecraft and Rockets, Vol. 38, No. 4, 2001, pp. 622-629.

[19] Oyama, T., Yamakawa, H. and Omura, Y., "Orbital Dynamics of Solar Sails for Geomagnetic Tail Exploration," Journal of Guidance, Control and Dynamics, Vol. 45, No. 2, 2008, pp. 316-323. doi: $10.2514 / 1.31274$

[20] De Juan Ovelar, M., Llorens, J. M., Yam, C. H. and Izzo, D., "Transport of Nanoparticles in the Interplanetary Medium," 60th International Astronautical Congress, Daejeon, Republic of Korea, 2009.

[21] Fortescue, P., Stark, J. and Swinerd, G. (eds.), Spacecraft System Engineering, Third Edition ed. Wiley, Chichester, 2003.

[22] Blitzer, L., "Handbook of Orbital Perturbationss," University of Arizona, 1970. 
[23] Cook, G. E., "Luni-Solar Perturbations of the Orbit of an Earth Satellite," Geophysical Journal International, Vol. 6, 1962, pp. 271-291.

[24] Kozai, Y., "Effects of Solar Radiation Pressure on the Motion of an Artificial Satellite," Smithsonian Contributions to Astrophysics, Vol. 6, 1963, p. 109.

[25] Aksnes, K., "Short-Period and Long-Period Perturbations of a Spherical Satellite Due to Direct Solar Radiation," Celestial Mechanics and Dynamical Astronomy, Vol. 13, No. 1, 1976, pp. 89-104.

[26] Escobal, P. R., Methods of Orbit Determination, R. E. Krieger Pub. Co., New York, 1965.

[27] Wolverton, R. W. (ed.), Flight Performance Handbook for Orbital Operations, ed. J.W.a. Sons, New York, 1963.

[28] King-Hele, D., Theory of Satellite Orbits in an Atmosphere, Butterworths, London, 1964.

[29] Vallado, D. A., Fundamentals of Astrodynamics and Applications, Third Edition, Space Technology Library, New York, 2007.

[30] Abramowitz, M. and Stegun, I. A., Handbook of Mathematical Functions, Applied Math. Series \#55, Dover Publications, 1965.

[31] Coleman, T. F. and Li, Y., "On the Convergence of Interior-Reflective Newton Methods for Nonlinear Minimization Subject to Bounds," Mathematical Programming, Vol. 67, No. 1, 1994, pp. 189-224.

[32] Coleman, T. F. and Li, Y., "An Interior Trust Region Approach for Nonlinear Minimization Subject to Bounds," SIAM Journal on Optimization, Vol. 6, No. 2, 1996, pp. 418-445.

[33] Dormand, J. R. and Prince, P. J., "A Family of Embedded Runge-Kutta Formulae," Journal of computational and applied mathematics, Vol. 6, No. 1, 1980, pp. 19-26.

[34] Petschek, H. E., Rayburn, C., Sheldon, R., Vickers, J., Bellino, M., Bevis, G. and Spence, H. E., The KiloSatellite Constellation Concept, Science Closure and Enabling Technologies for Constellation Class Missions, V.A.a.P. Panetta, University of California, 1998. 